\title{
Influence of guide vanes on the flow fields and performance of axial pump under unsteady flow conditions: Numerical study
}

\author{
Ahmed Ramadhan Al-Obaidi \\ Mustansiriyah University, Faculty of Engineering, Mechanical Engineering Department, Baghdad, Iraq. \\ Phone: +9647727547147
}

ABSTRACT - Influence of different guide vanes on structural of flow field and axial pump performance under unsteady flow is carried out using numerical method. A three-dimensional axial flow pump model is numerically simulated using computational fluid dynamics (CFD) method with four number of impeller blades and 3, 4, 5 and 6 guide vanes depend on the SIMPLE code, standard turbulence $k-\varepsilon$ model as well as sliding mesh method (SMM). The static, dynamic, total pressures, shear stress, velocity magnitude and turbulent kinetic energy are the important features which affecting instability operation in the pump. By monitoring above parameters and setting different measurement pressure points, the average pressures in the pump are discussed and the effect of guide vanes on the average pressure is analyzed. The results demonstrate that the numerical calculations can provide good accurately prediction for the characteristics of internal flow in the pump. The numerical results are closed to experimental results the minimum errors of pressure differences can reach $2.5 \%$ and the maximum errors $6.5 \%$. The guide vanes have more effect on the flow field and pressure variations especially at outlet region in the axial pump. As compared with the using various guide vanes, the pressure increases as number of vanes increase that can lead the performance of pump also increases. Pressure differences in the pump at variety mass flow for vane 6 is higher than other vanes 3,4 and 5 by $14.13,11.35$ and $3.85 \%$ for flow of 5 $\mathrm{L} / \mathrm{min}$. Further, the dynamic pressure differences for design flow between different vanes $6,5,4$ and 3 are about by $2.87,7.26$ and $8.51 \%$ respectively.
ARTICLE HISTORY

Revised: 24 $24^{\text {th }}$ Dec 2019

Accepted: 31st Dec 2019

\section{KEYWORDS}

Axial pump;

guide vane;

static pressure;

total pressure;

unsteady flow.

\section{INTRODUCTION}

Axial flow pump is commonly used in different types of applications such as municipal water supply, system of seawater desalinization [1], power plant generation, agricultural irrigation and ships [2,3]. the pressure fluctuations and fluid exciting vibration are the important issues in the turbo-machinery [4-6] such as various types of pumps and wind turbine [7, 8]. Different researchers were concentrated to investigate the flow at design conditions on performance of the pump $[9,10]$, but few investigations have focused to analyze the influence of guide vanes on global and local flow field analysis in the performance of a pump [11-16]. Experiment and numerical studied the structures of flow in the pump using guide blade was done by Toksoz [17] conducted the internal flow in the pump using adjustable guide vane. The results they have found hydraulic losses were increased, vortex produced within the diffuser and flow separation performed that leads necessity additional energy consumption and cause decreased pumping efficiency. The numerical results have shown that at the high pump the efficiency was slightly changed whereas the angle of vane setting was adjusted. Also, at off-design operating conditions using adjustable guide vanes the flow conditions can be improved, the efficiency can be increase and the hydraulic loss decrease.

Yi-bin [18] reported the unsteady fluid field inside the pump using guide vanes. Results noted that the fluctuation of pressure at the centre cross-sectional area in the inlet guide vane were firstly decreased then increased. Also, at the outlet guide vane region the pressure fluctuations were approximately symmetrically axial distribution. Moreover, at the middle suction of the vane was the minimum fluctuation of pressure amplitude was occurred. Another simulation studied the water flow in the axial pump was done by Qian [19]. They have observed that using guide vanes in the pump can decease the hydraulic losses and enhance the pump performance. Moreover, the results indicated that there are two important parameters to contribution the vortices and then cause the hydraulic losses in the channel of guide vane the first one was the attack angle and the second one was flow separation. Li [20] studied the influence of using guide vanes in the axial pump on flow field and Vibroacoustic features. They found that the vibration acceleration dominated frequency was BPF (blade passing frequency) is connected with characteristics of pressure pulsation. Moreover, as the rang of flow rate decreases the pressure fluctuating becomes very high. The results also revealed that when using nine vanes diffuser can decrease the amplitude of pressure fluctuations and vibration as compared to the other models. Yang [21] enhanced the axial pump efficiency under low flow rate conditions using mechanism of guide vanes. The results revealed that the fluid field in the axial pump by adjusting guide vane angle was improved. Also, the separation flow at the tail and inlet ledge of guide vane regions were reduced. Moreover, the results shown that the pumping hydraulic efficiency was also improved 
at off design conditions. Sang and Zhou [22] investigated the axial blood pump hydraulic performance under different outlet angle of guide vanes. They found that the pump performance such as efficiency and head were improved at the outlet angle guide vane equal $10^{\circ}$. When the outlet angle decreased that leads to the internal flow was more uniform and hence that can give higher pump head and efficiency. However, if outlet guide vane was very small that leads to increase the velocity within the guide vane channel and the cause more hydraulic loss in this region. Li et al. [23] investigated the influence of vane thickness parameter on fluctuations of pressure in a pump. Numerical results shown that the type of mesh, and turbulence model can effectively to predict the internal flow in the pump. The blade passing frequency of impeller was pressure fluctuation dominant frequency. Also, the results revealed that at the inlet of impeller the guide vane thickness has less effect on pressure fluctuation. But it has more effect at in the centre of guide vane region. Moreover, in order to obtained the stability in the internal flow of guide vane it can be decreased the guide vane thickness appropriately.

Kim et al. [24] analysed the influence of vane interactions in the pump designed. They found that when the number of an impellers blade increases the head also increases. In addition, when the guide vanes increase that leads to the flow interactions increase. Qian et al. and Al-Obaidi [25-28] evaluated the axial pump performance using adjustable guide vanes. the results shown that using guide vane can enhancement the pump efficiency that leads to improve the economic benefits in the hydropower applications. Song et al. [29] researched the influence of vortex flows in the pump. Results observed that at pressure amplitude reduced as of flow rate increased. At high flow the vortex increases into the impeller and hence the flow instability increasing causing more vibration in the pump. Song and Liu [30] conducted the effect of vortex flow on the axial pump performance. Results noted that the pressure was mostly influenced by pump impeller rotation speed. Also, the pressure in characteristic of time domain curves was changed as vortex increased. Zhou et al.[31] investigated pressure fluctuation in the axial pump. Results noticed that at the outer impeller diameter the pressure fluctuation has largest values and smallest values was occurred at the guide blade inlet.

The main aim of this numerical work is to enhance the performance of axial pump through modifying guide vanes of an axial impeller. Numerical method using CFD technique is implemented to analyze the mechanism of inner flow in the axial pump using different guide vanes by comparison of hydraulic flow to distinguish the guide blade adjustable advantages. In this current research, the effects of various number of guide vanes on field of flow such as pressure, velocity magnitude, turbulent kinetic energy, velocity, shear stress, and average pressure were analyzed by numerical methods in 3D unsteady of an axial pump, to provide more details for the researchers about the pump design and stable operation condition in such type of pumps.

\section{RESEARCH OF AN AXIAL FLOW MODEL}

To validate numerical of axial pump model, the results were compared with available experimental results from original test bench used by Mostafa and Mohamed [32]. To evade the effect of boundary conditions limits, to ensure the flow inside inlet pipe is fully developed and numerical stability results the computational domain at inlet and outlet pipe are extended as illustrated in Figure 1. Axial pump design flow parameters in this work are design mass flow is 12.5 $\mathrm{L} / \mathrm{min}$, impeller rotational of 3000 (rpm), impeller blades of four and guide vane number is also four. Three dimensional numerical pump model is created using Fluent (CFD technique). 3D models of whole model including inlet, outlet, axial impeller and guide vane are depicted in Figure 1.

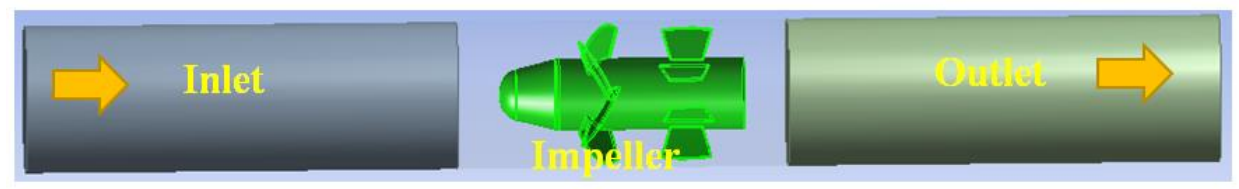

(a)

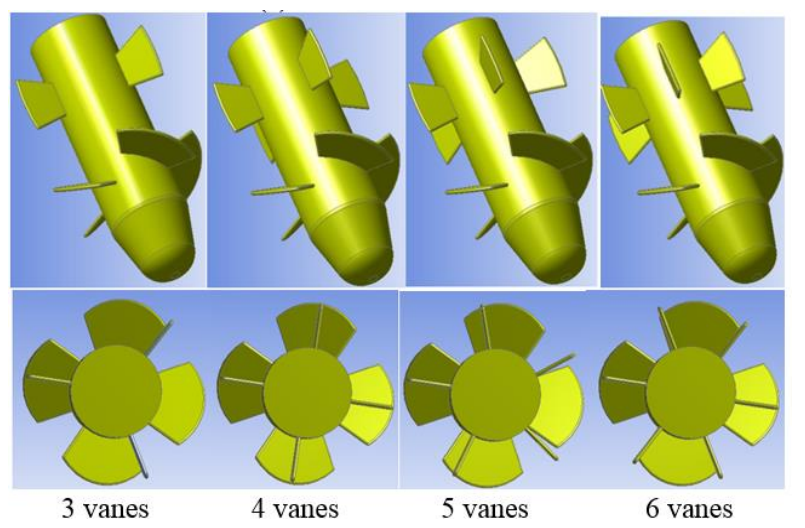

(b) 


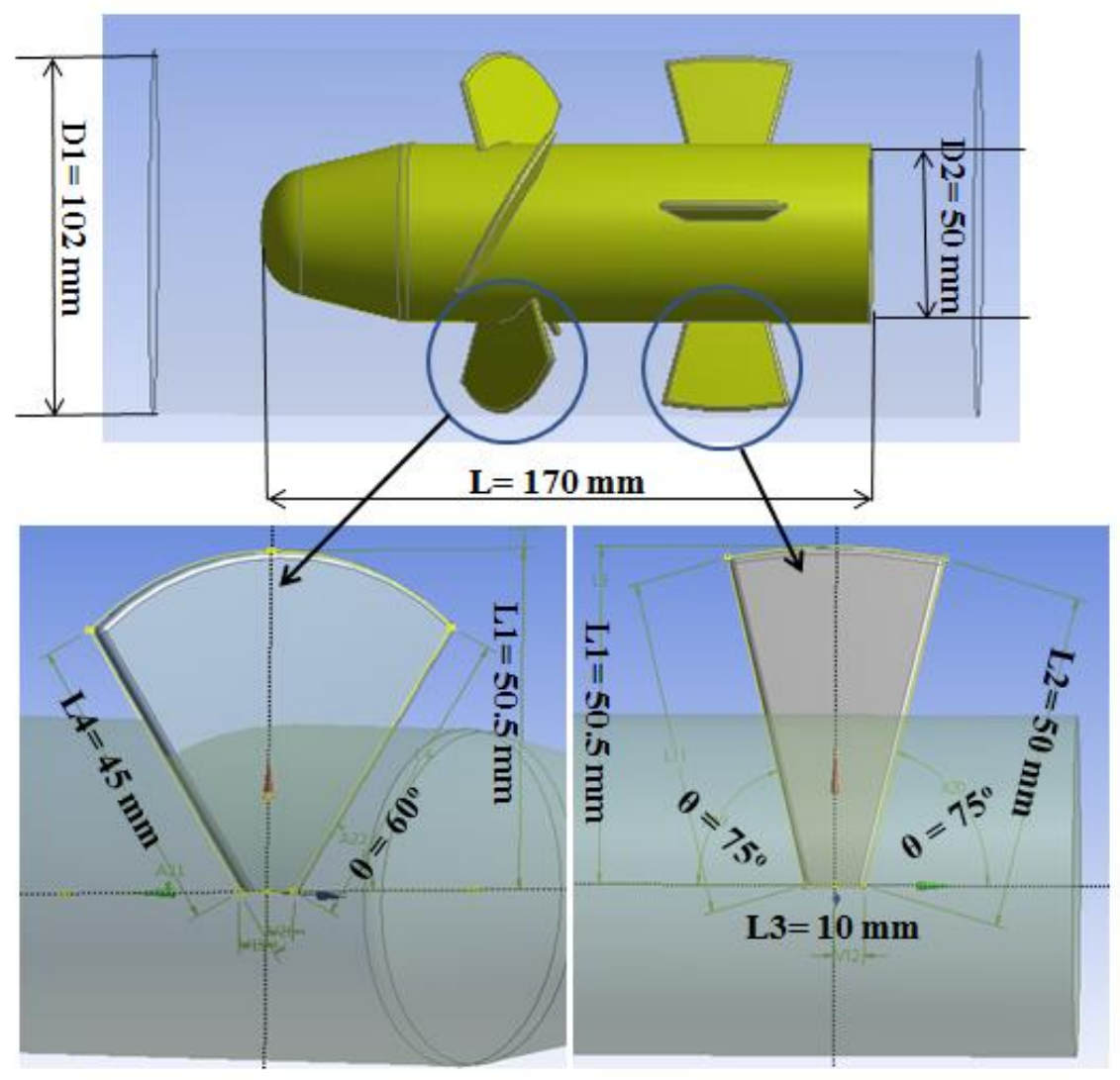

(c)

Figure 1. (a) 3D of whole pump model, (b) different impeller and impeller guide vane models and (c) the vane model dimensions.

In this work, to validate and compare the numerical result the available experimental data carried out by Mostafa and Mohamed [32] is chosen as research object. Table 1 summarized the specifications of experimental pump test.

Table 1. Specifications of axial pump test.

\begin{tabular}{ccc}
\hline Part & Value & Unit \\
\hline Design speed & 3000 & $(\mathrm{rpm})$ \\
Design Motor power & 2.2 & $(\mathrm{~kW})$ \\
Design flow rate & 12.5 & $(\mathrm{~L} / \mathrm{min})$ \\
Design head & 3 & $(\mathrm{~m})$ \\
Tip diameter & 102 & $(\mathrm{~mm})$ \\
Number of blades & 4 & $(-)$ \\
Hub diameter & 50 & $(\mathrm{~mm})$ \\
Hub to tip ratio & 0.495 & $(-)$ \\
Blade angles & 60 & $($ degree $)$ \\
Blade thickness & 2 & mm \\
\hline
\end{tabular}

\section{GRID GENERATION OF AN AXIAL FLOW MODEL}

Whole flow mesh domain in this study including inlet section part, axial impeller, guide vane part and outlet. unstructured meshing type (tetrahedral mesh) is used due to complicated computational domain structure in the internal flow domain and complex geometry of the axial pump. For computational domain interface mesh between impeller and guide vane the (ANSYS ICEM CFD) mesh is adopted and for critical regions in the flow field the local refinement is employed [33-35]. Under unsteady flow numerical simulation and at design operating condition the difference pressure is calculated then to minimize the effect of mesh size accuracy of computational calculated the mesh independent test is adopted and the mesh element two million is selected for numerical analysis. Figure 2 depicts the whole flow field meshing domain for an axial flow pump, inlet and outlet parts. The mesh size is $1 \mathrm{~mm}$ and the number of nodes are 3 million. Then mesh independent testing is compared in order to obtain more accuracy simulation results. So, three meshes 
one, two and three million are used the three million mesh generation for flow domain is chosen for analyzing in this work.

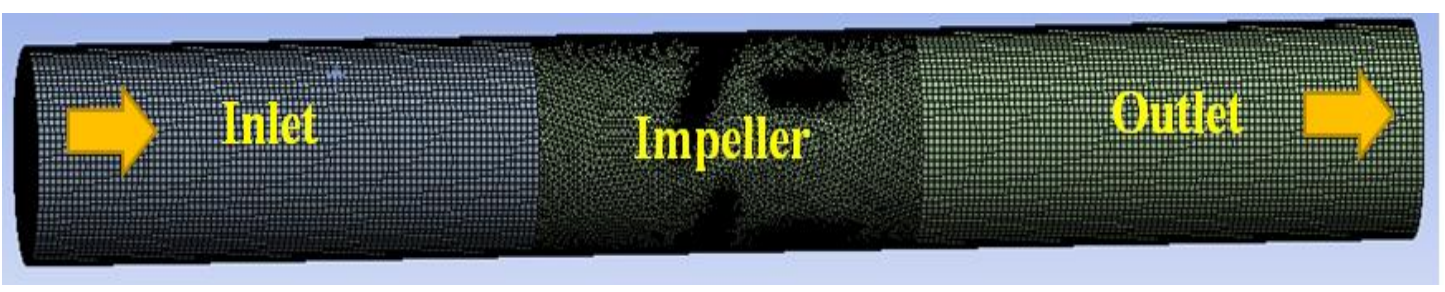

(a) Three-dimensional of whole axial pump domain meshing

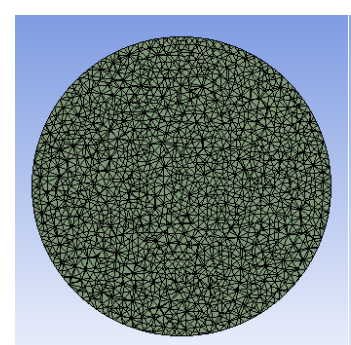

Pipe

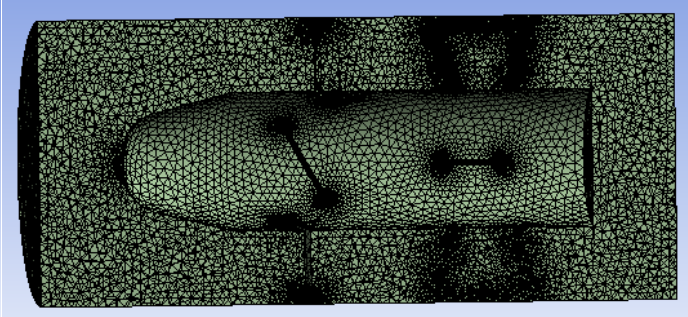

Impeller

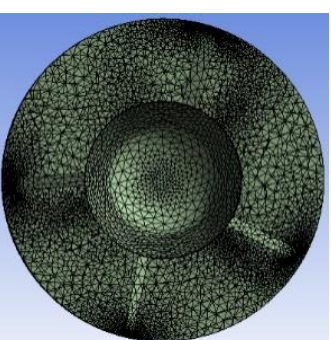

Guide vane

(b) Meshing for pipe domain, axial impeller and vanes

Figure 2. (a) Three-dimensional of whole axial pump and (b) different mesh domains.

\section{Governing Equations}

The turbulent incompressible flow governing equations are the RANS Equations for the momentum and mass conservation presented as [36]:

$$
\begin{gathered}
\frac{\partial}{\partial x_{i}}\left(\rho \bar{u}_{i}\right) \\
\frac{\partial}{\partial x_{i}}\left(\rho \bar{u}_{i} \bar{u}_{j}\right)=-\frac{\partial \bar{P}}{\partial x_{i}}+\frac{\partial}{\partial x_{j}}\left[\mu \frac{\partial \bar{u}_{i}}{\partial x_{j}}-\rho \bar{u}_{i} \bar{u}_{j}\right]
\end{gathered}
$$

where, $\bar{P}$ and $\mu$ are denoted the averaged pressure parameter and molecular viscosity. $-\rho \bar{u}_{i} \bar{u}_{j}$ represented the function of Reynolds stress.

\section{Standard Turbulence k- $\varepsilon$ Model}

This kind of turbulence model was usually used in different types of flow field analysis applications such as pumps and turbines. The k- $\varepsilon$ equations consist of different terms, for function of turbulent kinetic energy [36] can be calculated using below equation.

$$
\frac{\partial(\rho k)}{\partial t}+\frac{\partial\left(\rho k u_{i}\right)}{\partial x_{i}}=\frac{\partial}{\partial x_{j}}\left[\frac{\bar{\mu}_{t}}{\sigma_{k}} \frac{\partial k}{\partial x_{i}}\right]+2 \mu_{t} E_{i j}-\rho \epsilon
$$

For calculation dissipation using below equation:

$$
\frac{\partial(\rho \epsilon)}{\partial t}+\frac{\partial\left(\rho \epsilon u_{i}\right)}{\partial x_{i}}=\frac{\partial}{\partial x_{j}}\left[\frac{\bar{\mu}_{t}}{\sigma_{\epsilon}} \frac{\partial \epsilon}{\partial x_{i}}\right]+C_{1 \epsilon} \mu_{t} E_{i j}-C_{2 \epsilon} \rho \frac{\epsilon^{2}}{k}
$$

\section{NUMERICAL SIMULATION AND BOUNDARY CONDITIONS}

3D incompressible RANS equation is employed using Computational Fluid Dynamics to depicts the flow fields in an axial pump with SIMPLE computational technique. At near wall region, the standard wall surface is applied. The 
boundary operating conditions set at inlet part was velocity. The outlet parts the outflow [36]. Transient numerical calculation is carried out time step in this numerical simulation is set as $3.4482 \times 10^{-4} \mathrm{~s}$. The simulation results are need to 120 time steps in order to rotate for each circle at every impeller time step rotates $3^{\circ}$.

\section{EXPERIMENTAL AND NUMERICAL VALIDATION RESULTS}

Figure 3 depicts the characteristic of overall pump performance curves obtained by axial pump numerical and experimental results. Pressure differences across the pump (at inlet and outlet parts) using CFD technique are collected to validate and compare with experimental data. As it is observed that the numerical pressure differences are slightly higher than experimental available data. The numerical results are closed to experimental results the minimum errors of pressure differences can reach $2.5 \%$ and the maximum errors was around $6.5 \%$. These errors occur because of several reasons due to the mesh quality or complex pump geometry and flow cavitation especially at high flow rate. Generally, the overall numerical pump performance for pressure differences is good agreement with measurement test performance that leads to the mesh, turbulence model and numerical calculation setup in this study can provide good accuracy predication for the internal flow and characteristic the pump performance.

\section{FLOW PATTERN ANALYSIS}

There are different high interaction firstly occurs between the impeller and pipe wall and secondly happened between the axial impeller and various vanes. Figure 4 represents the change process of static pressure variations under changed range of mass flow namely 5, 10, 12.5, 17.5 and $20(\mathrm{~L} / \mathrm{min})$ and four guide vanes including 3, 4, 5 and 6 at same impeller speed is 3000 (rpm).

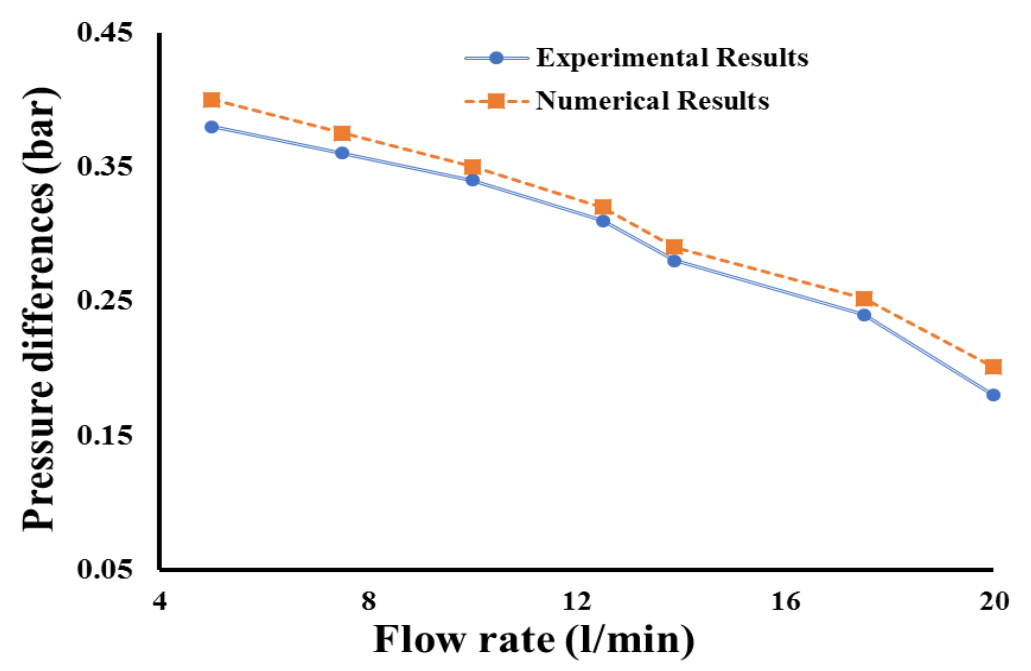

Figure 3. Experimental available date and numerical validation results.

As it is observed that when rotating of axial impeller that leads to occur two regions first one is formed higher pressure region near outlet impeller pressure surface. The second zone is formed low pressure area closed to inlet impeller suction part at this region when the pressure deceases below vapor water pressure can cause occurrence of cavitation in this area due to low pressure area then that causes more unstable internal flow and growth the noise and vibration. Second important pressure area is between both impeller and guide vanes, it can be noticed that when the axial impeller rotating that leads to cause the internal flow field disorder in the annular space between impeller outlet and inlet of vane space due high interaction. The pressure pulsation periodic emerges in the internal flow at the impeller outlet due to the static space guide vane interference to flow field outlet. Moreover, under different guide vanes the high-pressure region increase with vane increases due to increase guide vanes cause more interaction and hence that leads to increase the pressure at outlet the pump then increases the performance the pump. Besides, the maximum static pressure in this figure for design flow between different vanes 6, 5, 4 and 3 are around by 4.01, 5.66 and $6.74 \%$ respectively. 


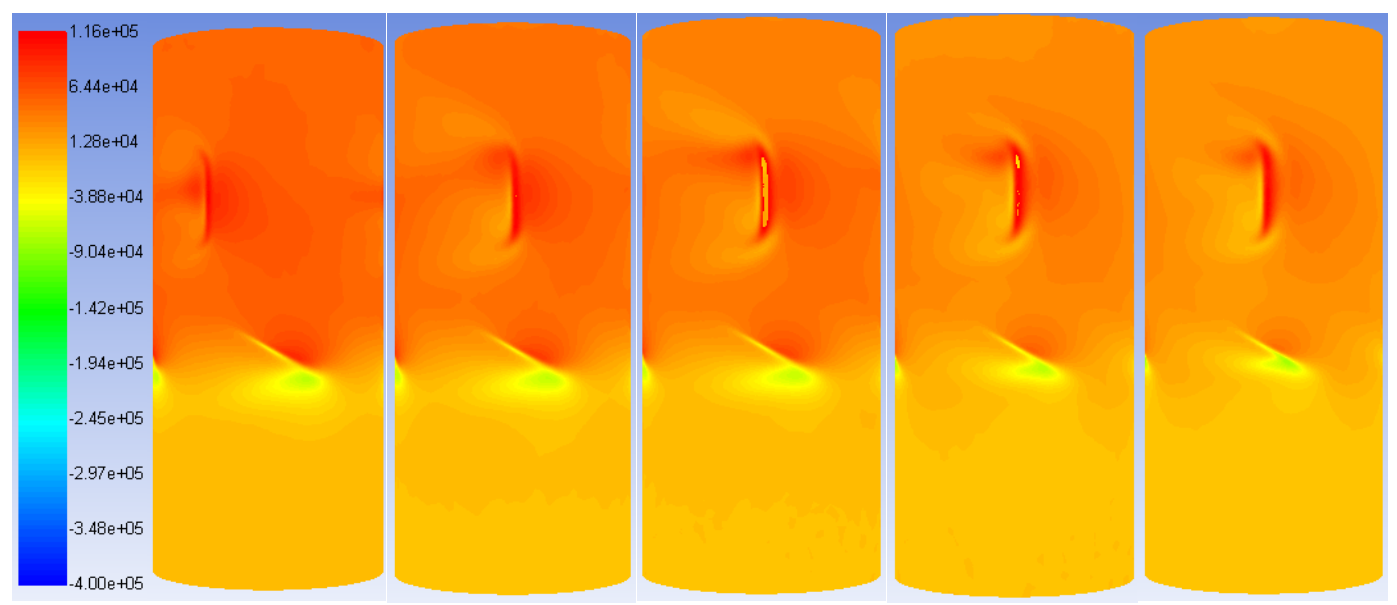

(a) Guide Vanes 3

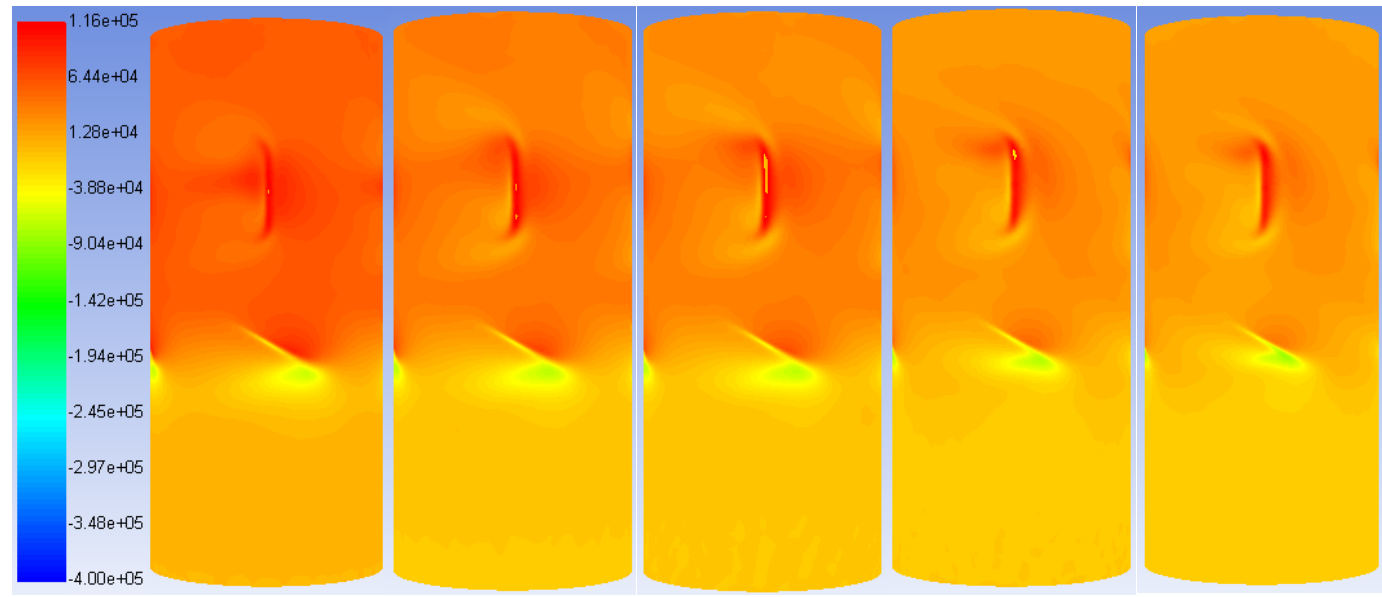

(b) Guide Vanes 4

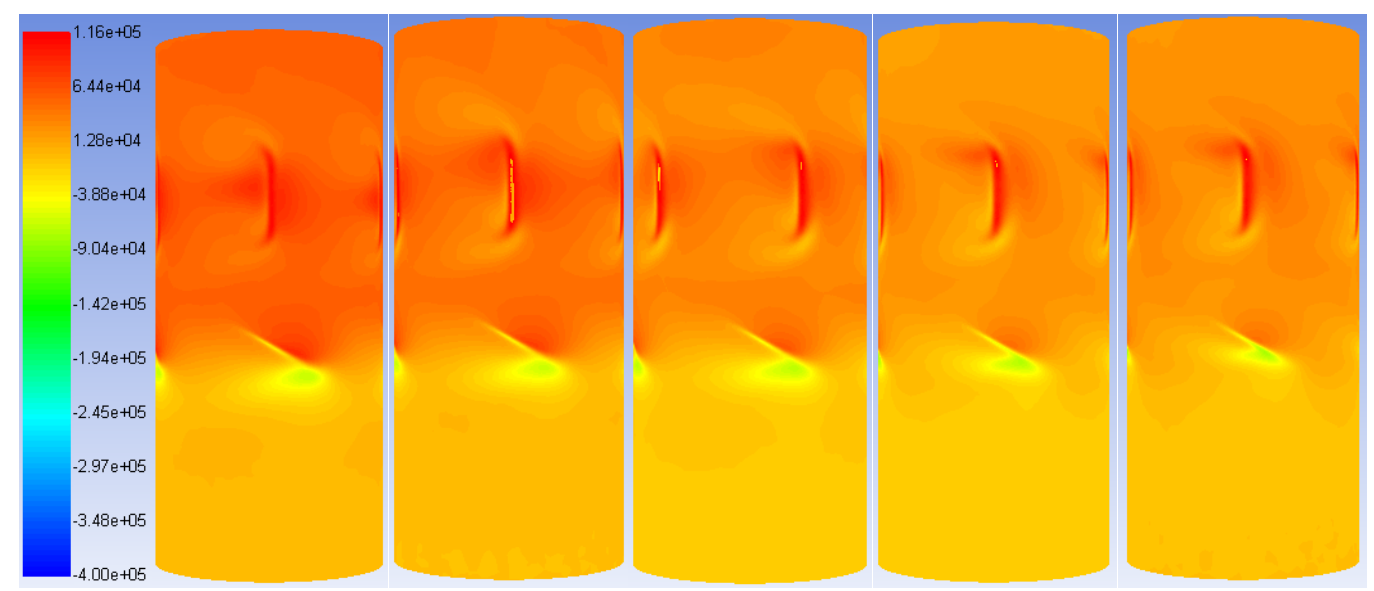

(c) Guide Vanes 5 


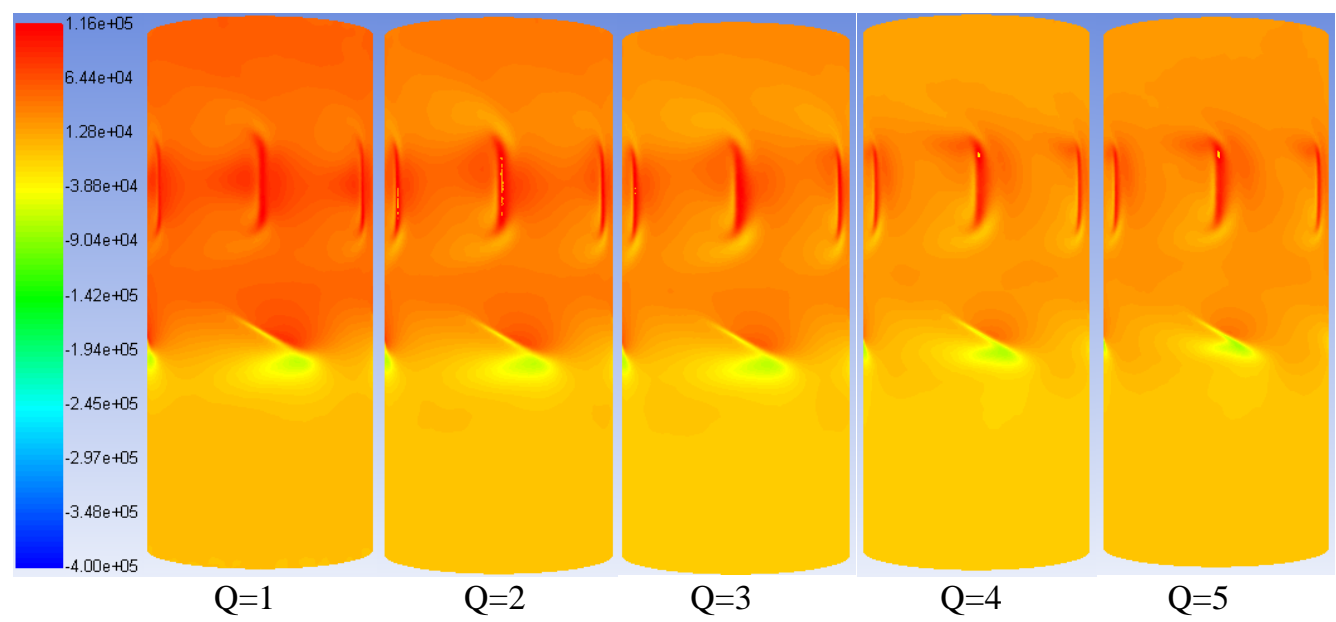

(d) Guide Vanes 6

Figure 4. Distribution of static pressure (Pa) in the pump at various flow rates and guide vanes.

Figure 5 describes distribution in dynamic pressure in a pump at various flow rates conditions and guide vanes. It is noticed that high dynamic pressure variations occur between two important regions first one between the outlet impeller and inlet vanes and the second one at outlet of the vanes region. Also, it can be observed that when the guide vanes increase that leads the flow passages between the vanes is narrowed and hence the internal flow velocity increases. Moreover, the important flow field disorder occurs at the annular space between outlet of axial impeller and inlet guide vanes. However, the pressure fluctuation change because of the interaction internal flow in the pump is mostly the similar trend over time. Further, the maximum dynamic pressure for design flow between different vanes $6,5,4$ and 3 are about by $2.87,7.26$ and $8.51 \%$ respectively.
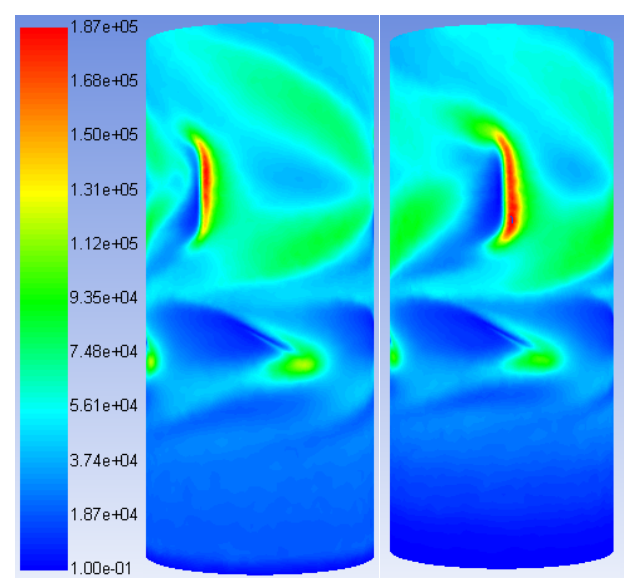

(a) Guide Vanes 3
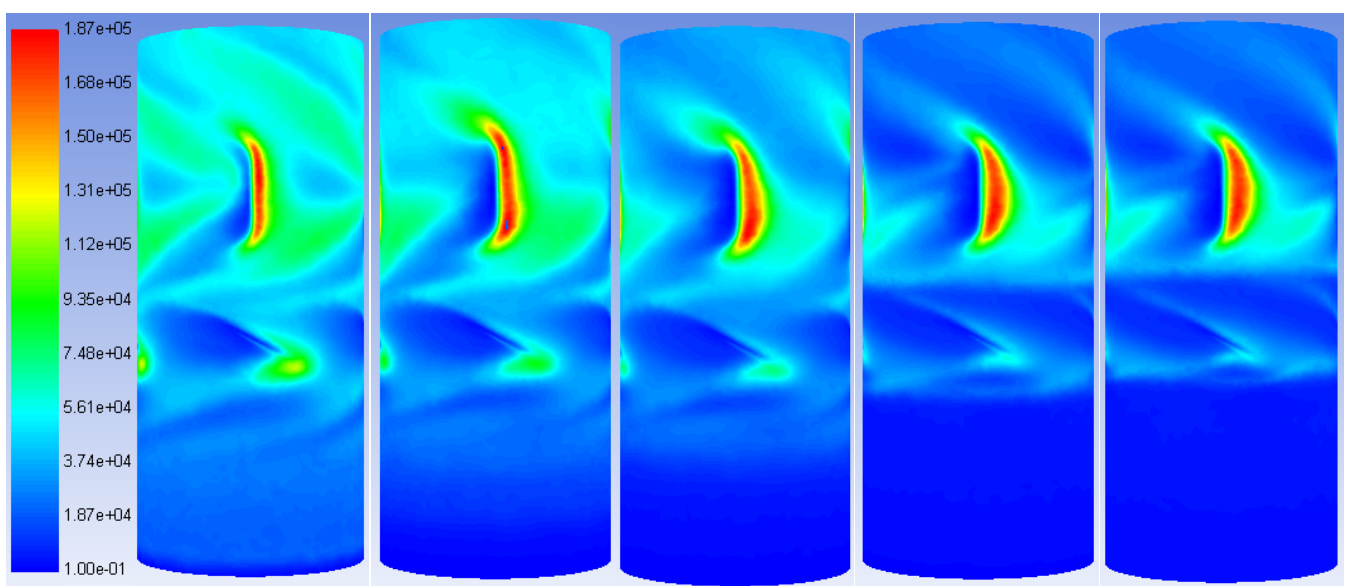

(b) Guide Vanes 4
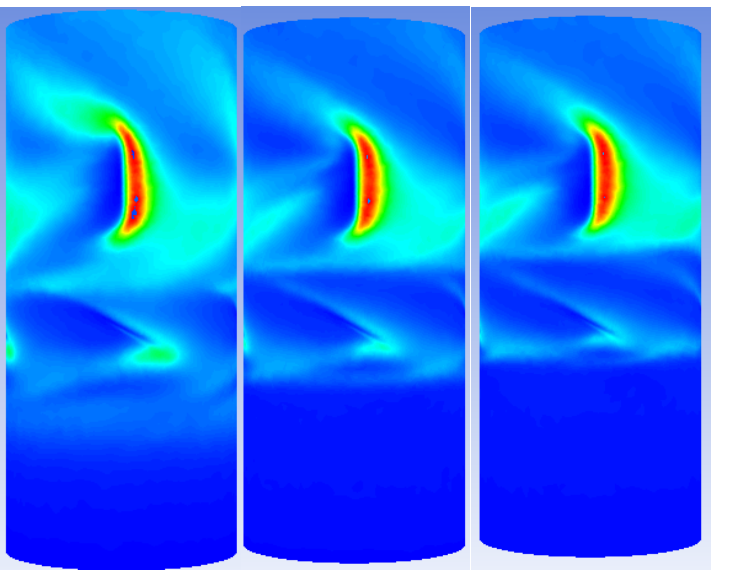


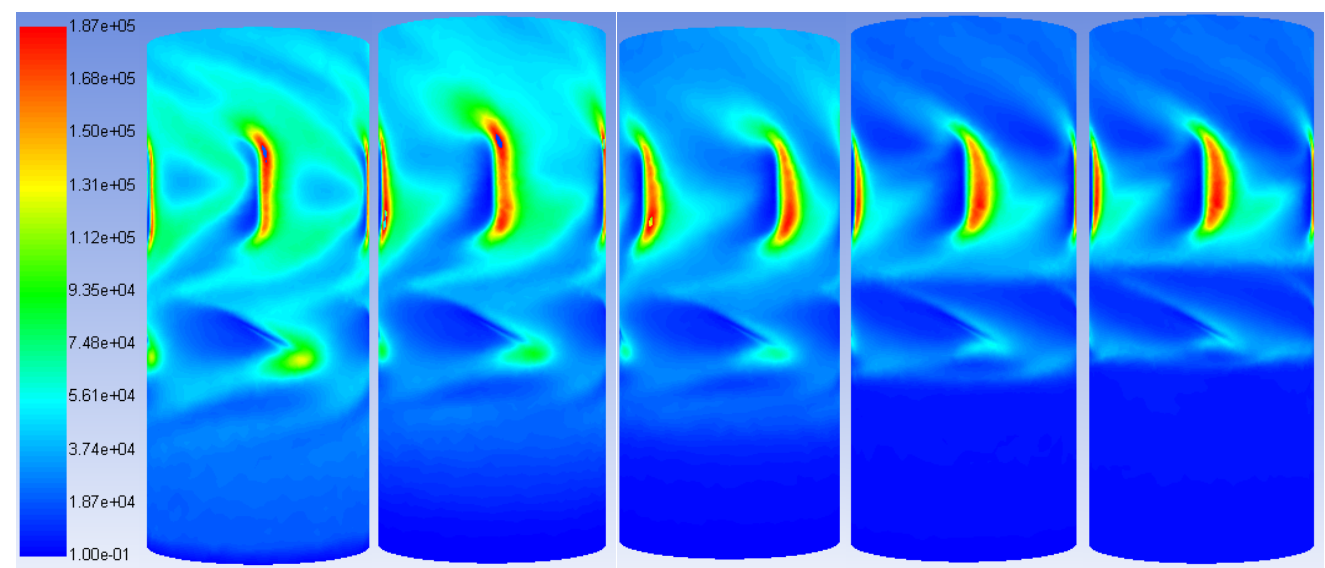

(c) Guide Vanes 5

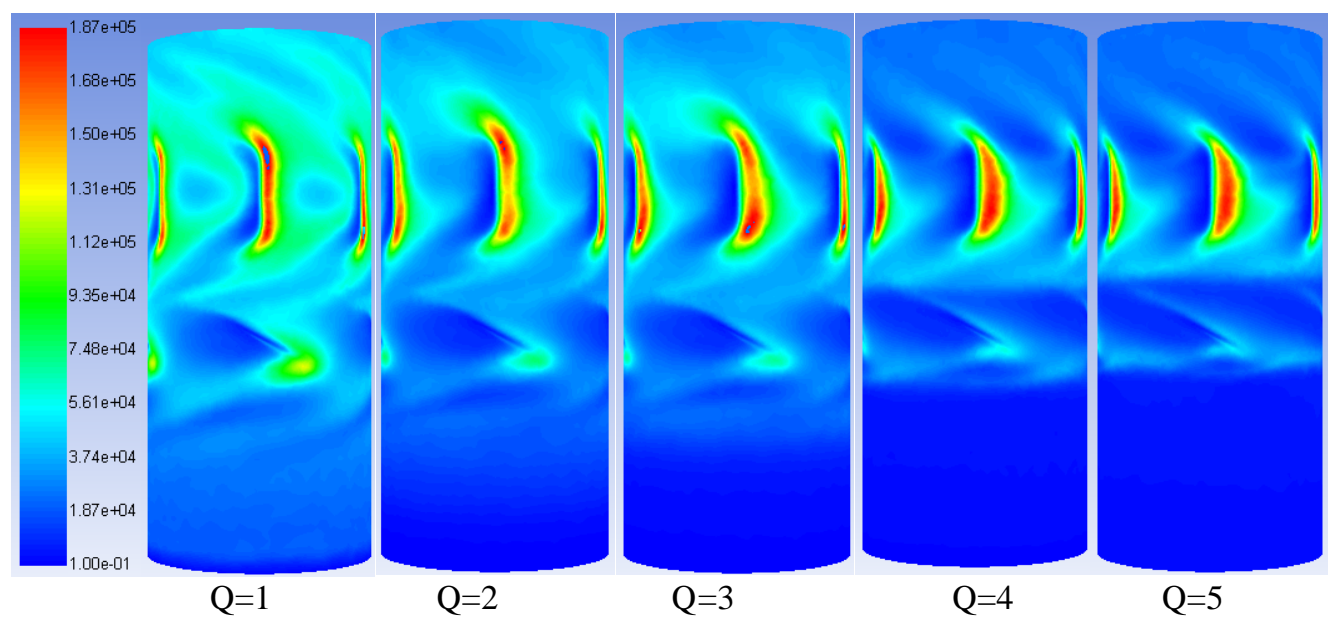

(d) Guide Vanes 6

Figure 5. Distribution of dynamic pressure ( $\mathrm{Pa}$ ) in the pump at various flow rates and guide vanes.

For more qualitative investigation regarding the influence of different guide vanes on total pressure in the pump, Figure 6 depicts variations in total pressure of an axial flow pump at several range of flow rates and guide vanes. It is noticed that the total pressure variations have the same trend static pressure variations. The high total pressure occurs at distance between the impeller and vanes and the total pressure is become more and more close to the tip vane region. Additionally, the numerical results revealed that pressure reduces when range of mass flow in the pump was increased. Furthermore, pressure was increased as guide vane increases. Therefore, it can be found that the add guide vanes have high influence on the pressure variations and overall of the pump performance. Furthermore, the maximum total pressure for design flow for vane 6 is higher than other vanes 5,4 and 3 by about $0.77,1.15$ and $2.58 \%$ respectively.

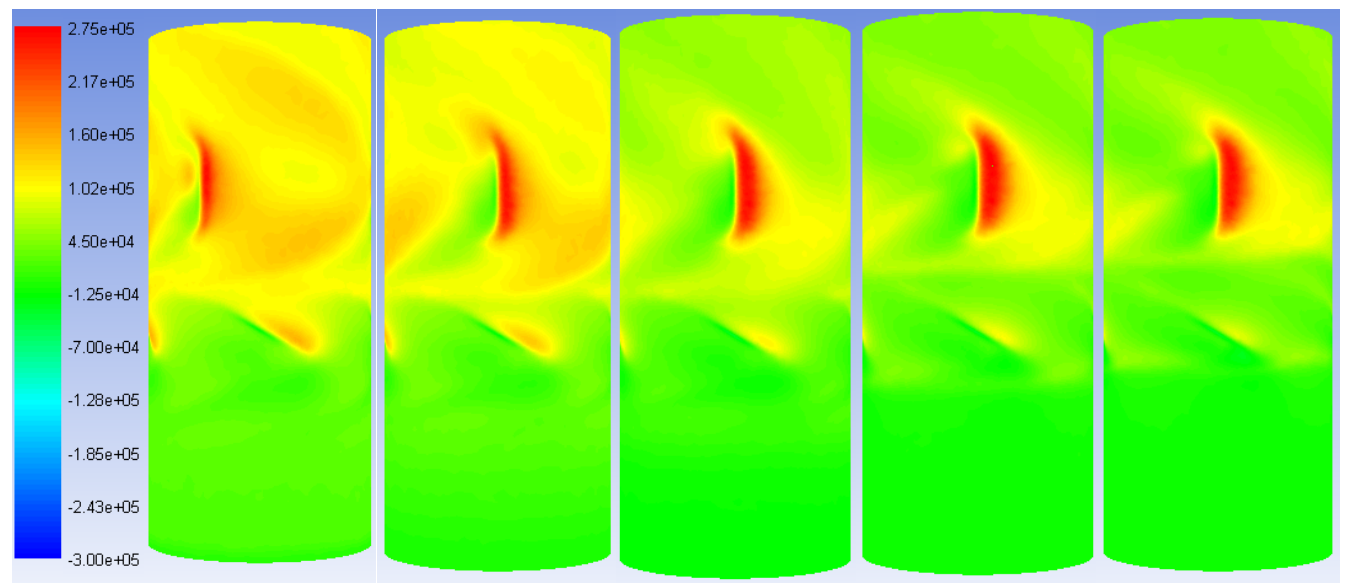

(a) Guide Vanes 3 


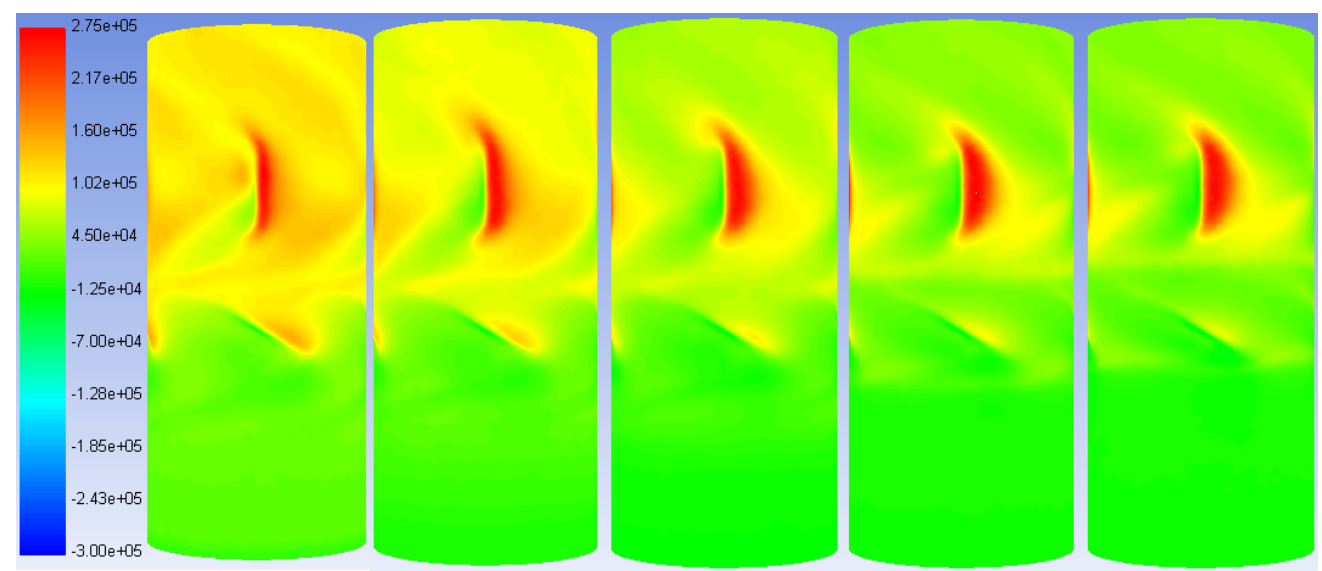

(b) Guide Vanes 4

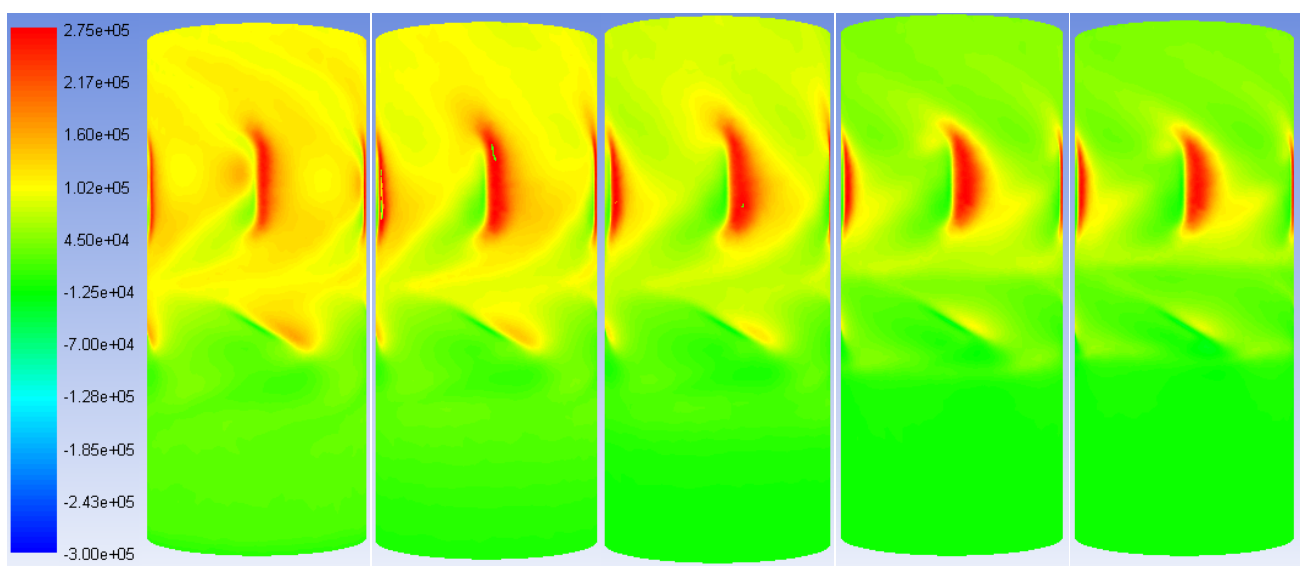

(c) Guide Vanes 5

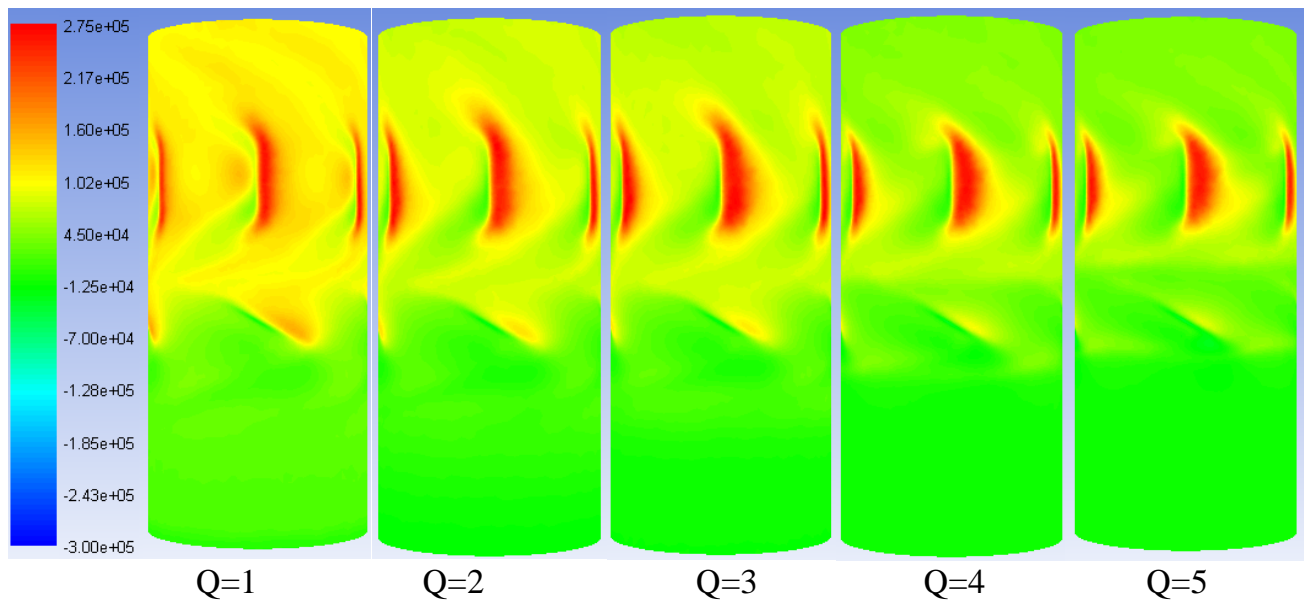

(d) Guide Vanes 6

Figure 6. Distribution of total pressure $(\mathrm{Pa})$ inside the pump.

Figure 7 depicts quantitative analysis the effect of different guide vanes, it can be clearly observed that when the vane increases that leads to the pressure differences across the pump also increases and hence that leads to performance of the pump increases. From above numerical analysis it can be coucluded that the guide vane has important effect on overal internal flow field and performance the pump. Moreover, pressure differences in the pump at variety mass flow for vane 6 is higher than other vanes 3,4 and 5 by $14.13,11.35$ and $3.85 \%$ for flow of $5 \mathrm{~L} / \mathrm{min}$ as presented in Table 2 for different mass flows. 


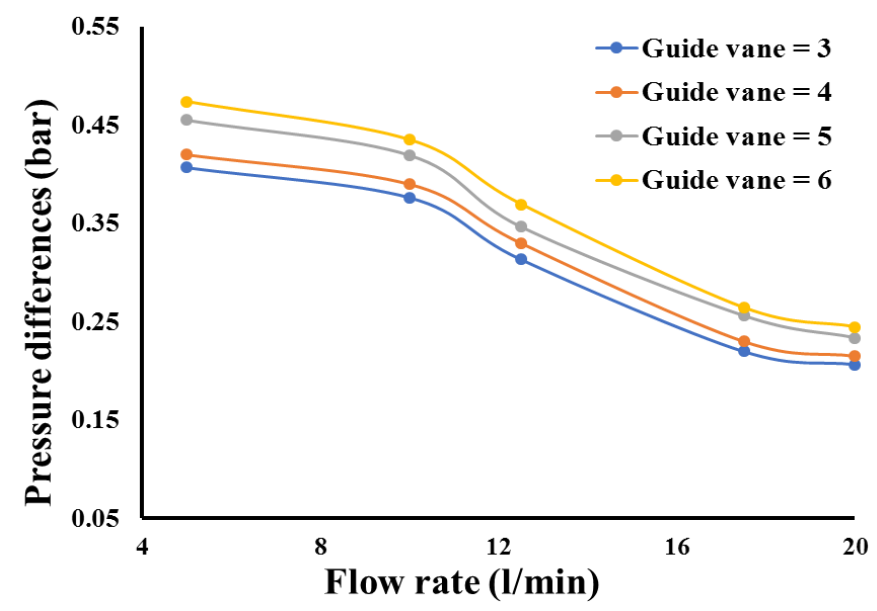

Figure 7. The influence of different blade vanes on the pump performance.

Table 2. Percentage different for pressure differences.

\begin{tabular}{|c|c|c|c|}
\hline $\begin{array}{l}\text { Flow } \\
\text { (L/min) }\end{array}$ & $\begin{array}{c}\text { vane } 6 \text { and } 3 \\
(\%)\end{array}$ & $\begin{array}{c}\text { vane } 6 \text { and } 4 \\
(\%)\end{array}$ & $\begin{array}{c}\text { vane } 6 \text { and } 5 \\
(\%)\end{array}$ \\
\hline 5 & 14.13 & 11.35 & 3.85 \\
\hline 10 & 13.50 & 10.34 & 3.52 \\
\hline 12.5 & 15.17 & 10.69 & 3.18 \\
\hline 17.5 & 16.74 & 12.95 & 4.10 \\
\hline 20 & 15.55 & 12.13 & 4.58 \\
\hline
\end{tabular}

\section{TURBULENT KINETIC ENERGY AND TURBULENT INTENSITY ANALYSES}

For more qualitative results in the pump, Figure 8 represents the turbulent kinetic energy variations. The results found that from this figure when the mass flow increases the turbulent kinetic energy (TKE) variations decreases and the high area of TKE happened at impeller tip blade and at tip guide vane blades. The reason behind that is due high interaction surfaces in these especial regions. Moreover, it can be notice that when the guide van increase that leads the turbulent kinetic energy variations also increases. That happens due to the high-pressure area occurs between the axial impeller area and guide vanes as well as at outlet vanes obviously increase with the vanes increase. Furthermore, the maximum TKE for design flow between different vanes $6,5,4$ and 3 are about by $0.02,0.19$ and $2.35 \%$ respectively.

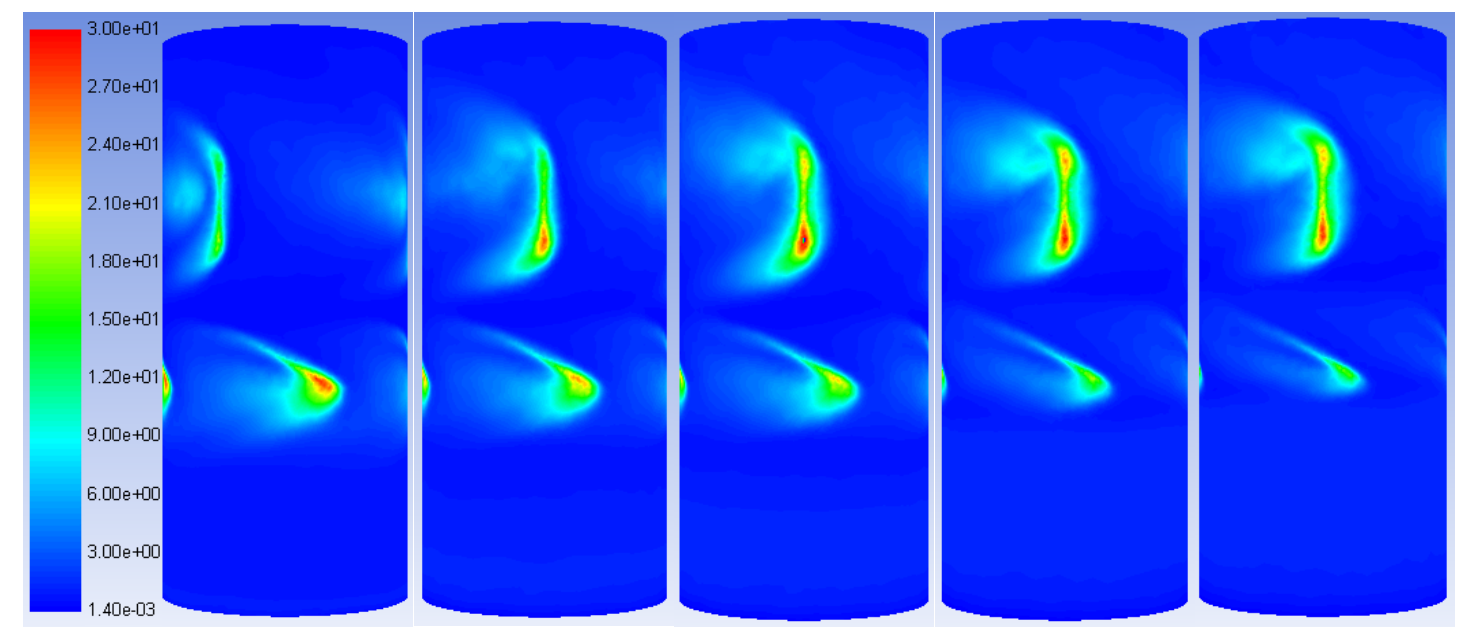

(a) Guide Vanes 3 

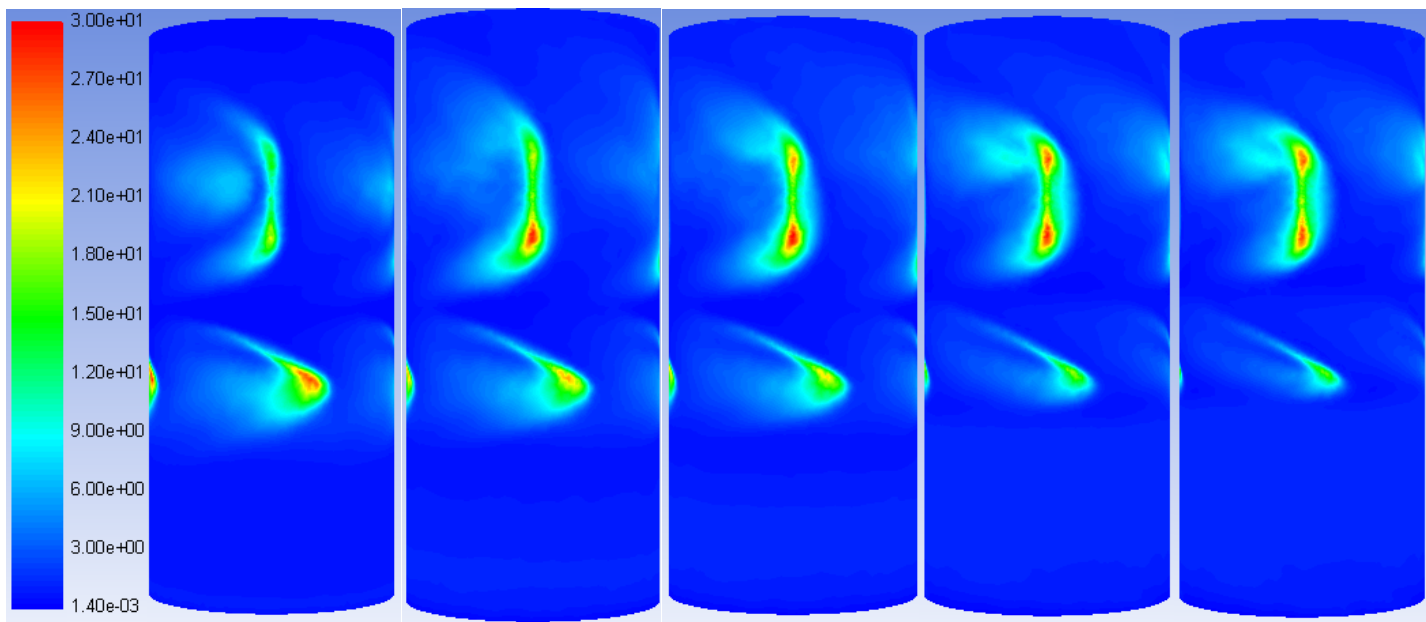

(b) Guide Vanes 4
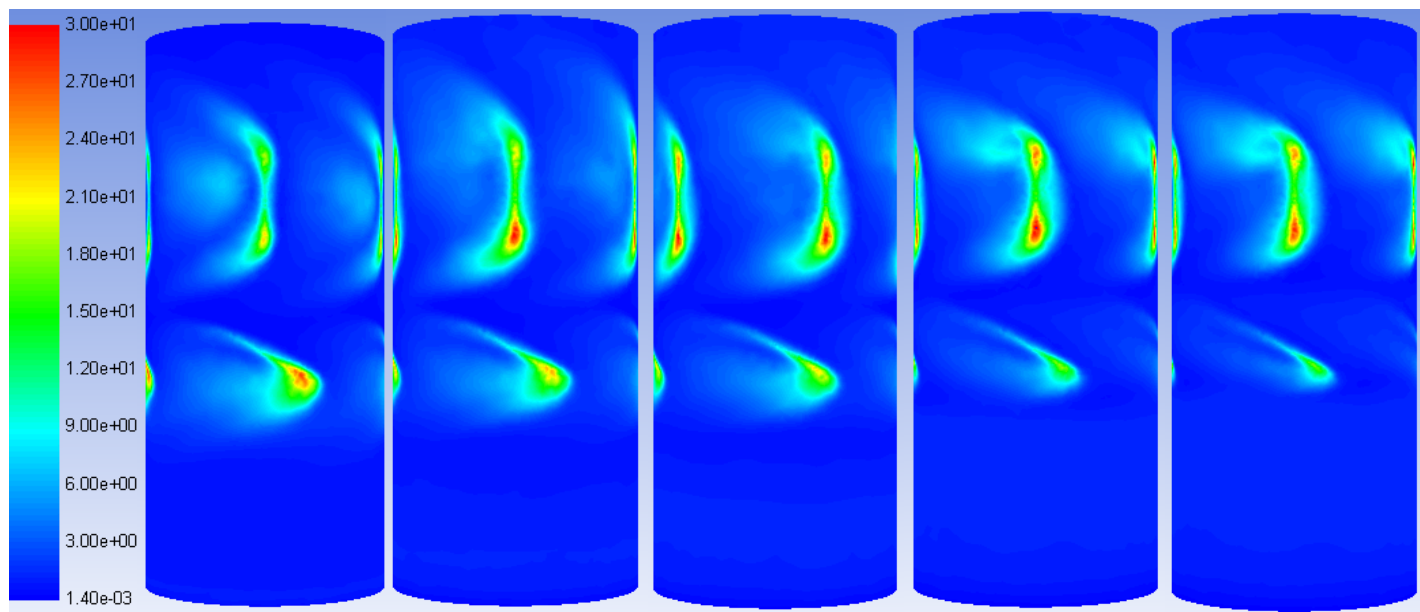

(c) Guide Vanes 5

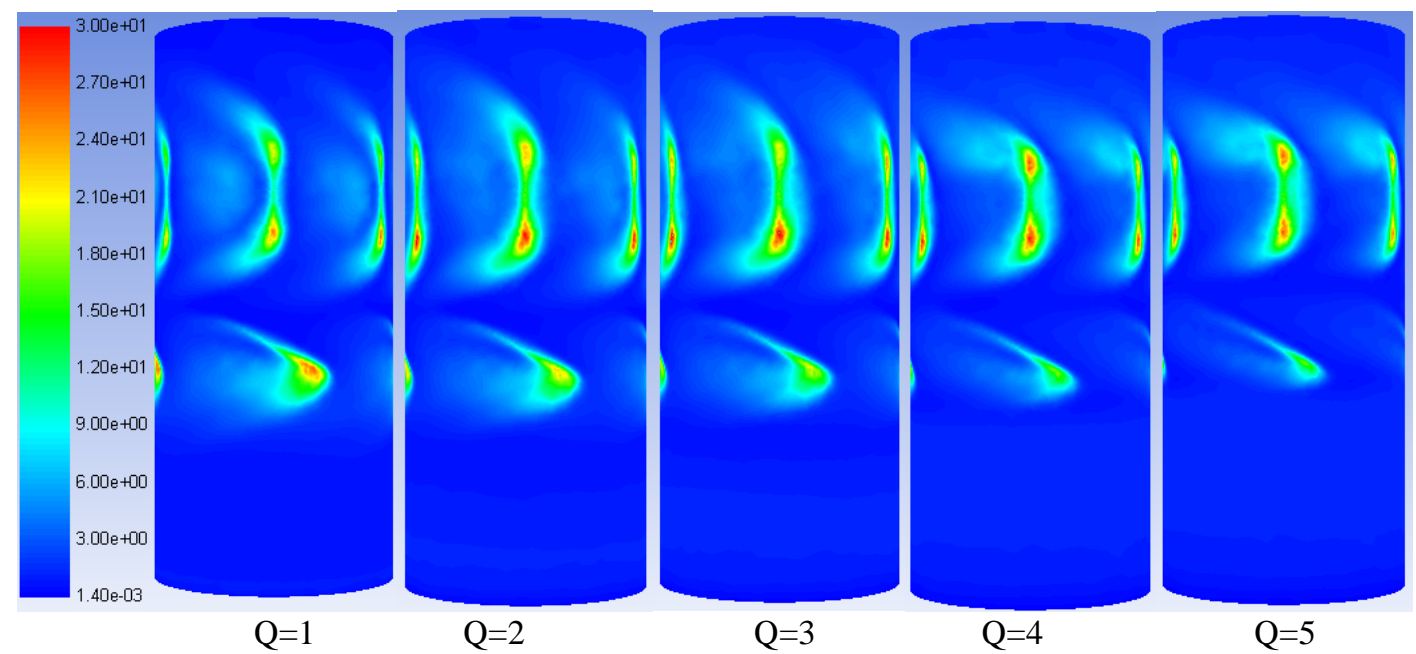

(d) Guide Vanes 6

Figure 8. Distribution of turbulent kinetic energy $(\mathrm{J} / \mathrm{kg})$ in the pump at various flow rates and vanes.

Figure 9 depicts variations in the turbulent intensity, it can be seen that the high region in turbulent intensity occurs at impeller blades and guide vanes and maximum value of turbulent intensity happened closed the region of tip blades for both impeller and vanes due to the same above reason in the last figure (Turbulent kinetic energy variations). Also, it can be observed that the turbulent intensity variations increase as guide vanes increases for all cases under investigations. That happen due to the impeller and vane complex structure as well as the disturbance flow can cause more vortex region can cover the axial impeller and the all guide vane passages. Again, it can be can concluded that guide vanes have high 
impact on flow in the axial pump. Furthermore, the maximum turbulent intensity for design flow between different vanes $6,5,4$ and 3 are about by $0.03,0.11$ and $1.32 \%$ respectively.

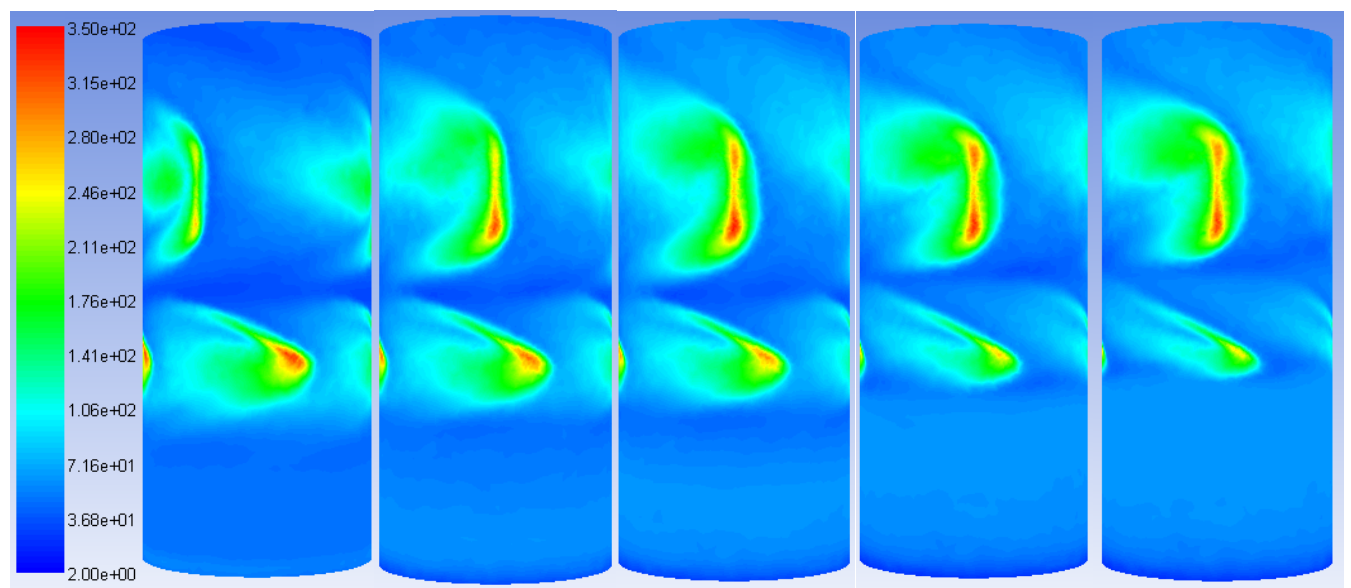

(a) Guide Vanes 3

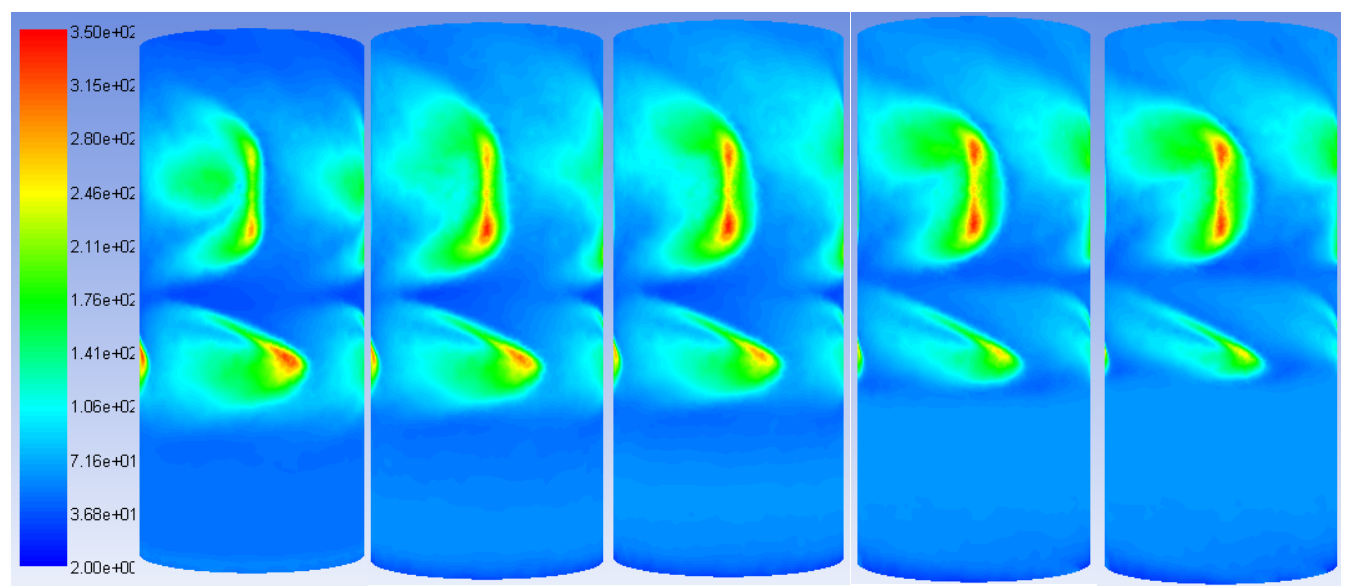

(b) Guide Vanes 4

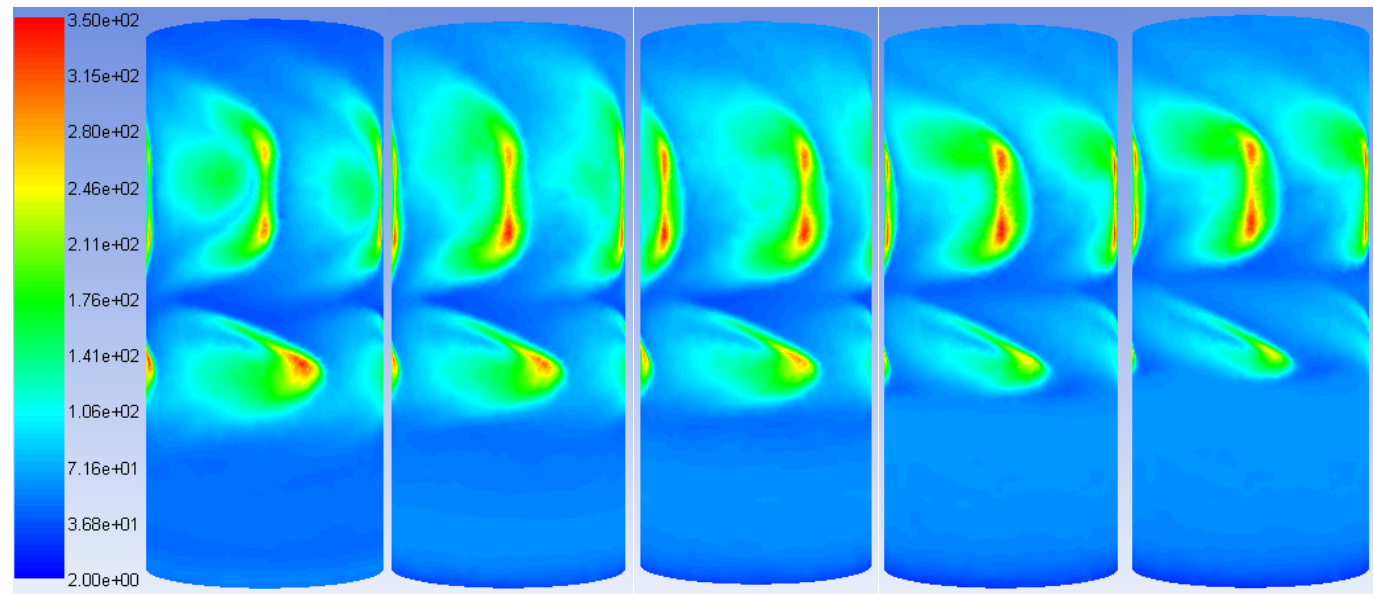

(c) Guide Vanes 5 


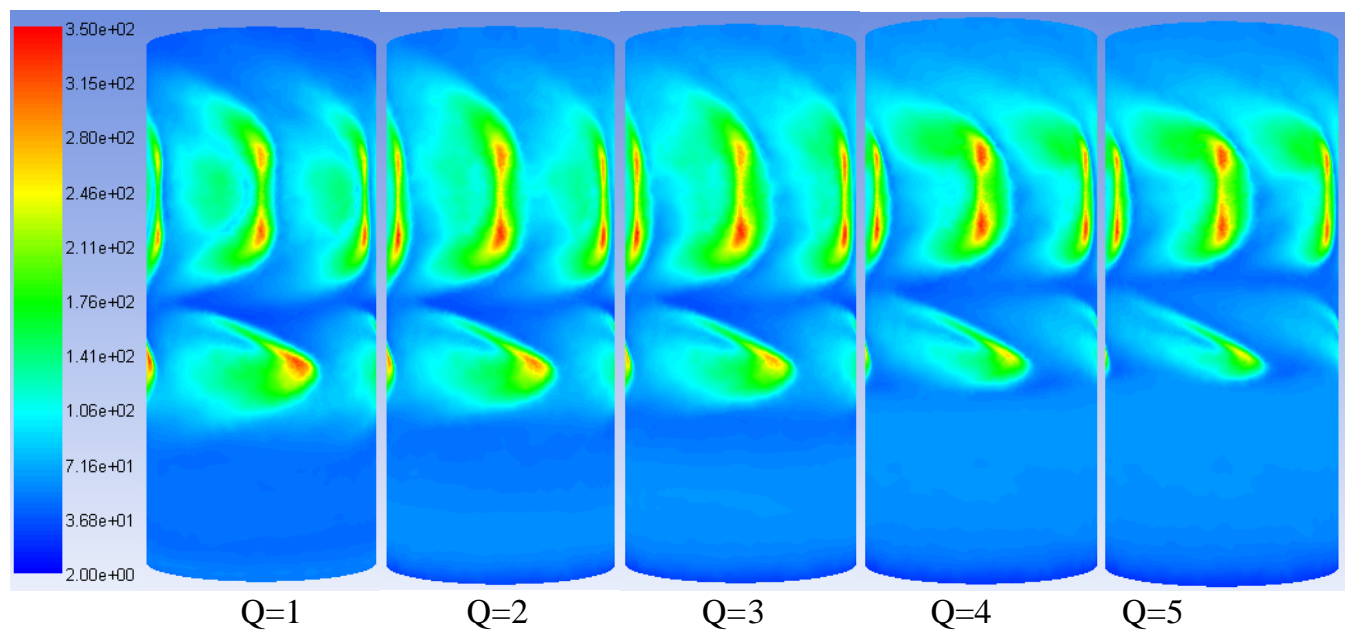

(d) Guide Vanes 6

Figure 9. Turbulent Intensity in a pump.

\section{ANALYSIS OF WALL SHEAR STRESS}

Numerical calculations in the pump are carried out using various guide and the mechanism of inner fluid can be investigated according to the numerical results. Figure 10 demonstrates variations in shear stress fields of the pump. As observed that flow fields (shear stress) round and near the impeller blades and guide vanes are in disorder state. Also, the maximum shear stress is at outlet impeller blades. The state of disorder for guide vans is higher than around axial impeller. The more section is effect closed the area of tip blades. The reason behind that is due to the effect of formation of vortex at the impeller blade and the back as well as at the tail of the vanes regions. Moreover, due to the flow separation which occurs especially at range of low flow rate. Additionally, it is noticed that the shear stress variations increase as guide vanes increase. Furthermore, it can be observed that at the pump outlet especially around and near the impeller and guide vanes a high rotational component in the flow was found and hence that leads to cause stronger shear stresses. Moreover, the maximum shear stress for design flow between different vanes $6,5,4$ and 3 are about by $3.47,4.07$ and $4.76 \%$ respectively.
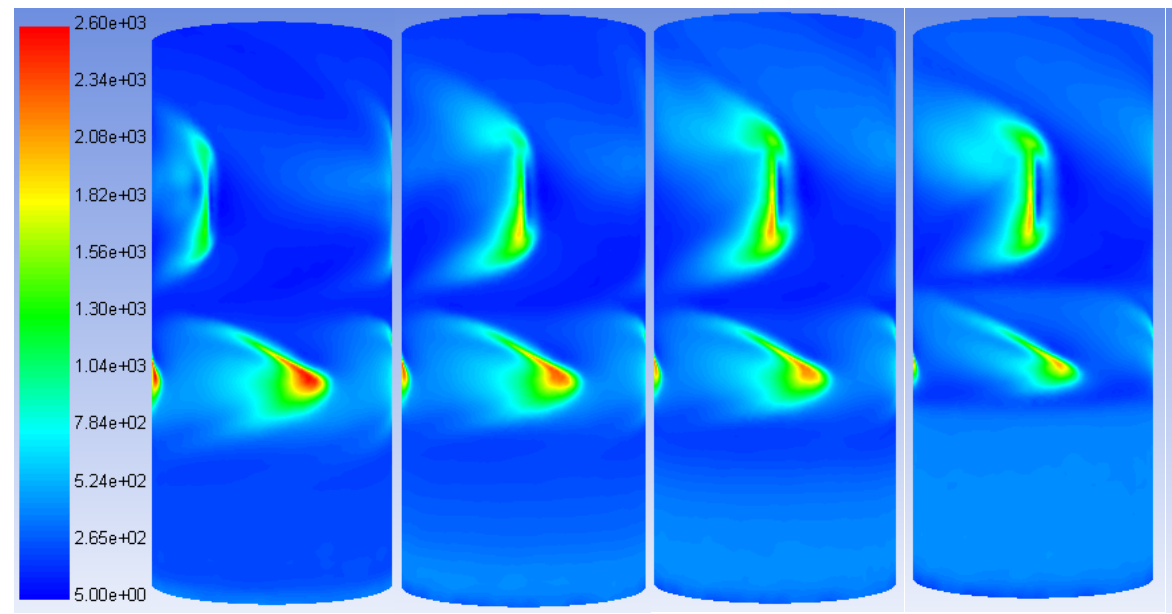

(a) Guide Vanes 3

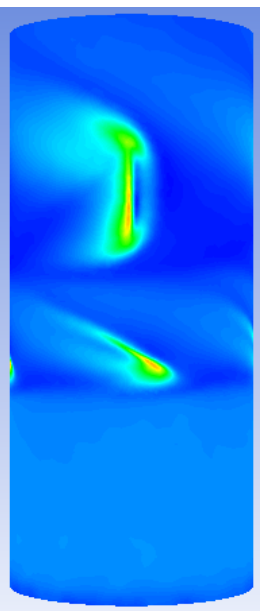

. 

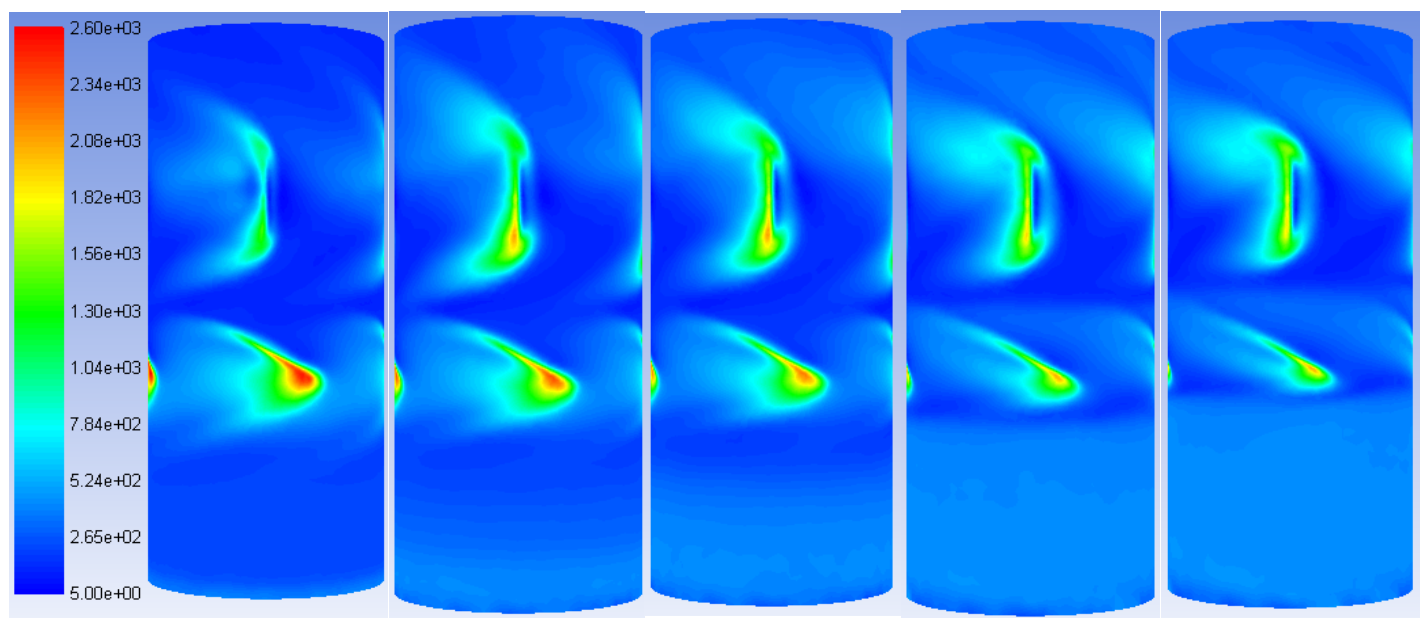

(b) Guide Vanes 4
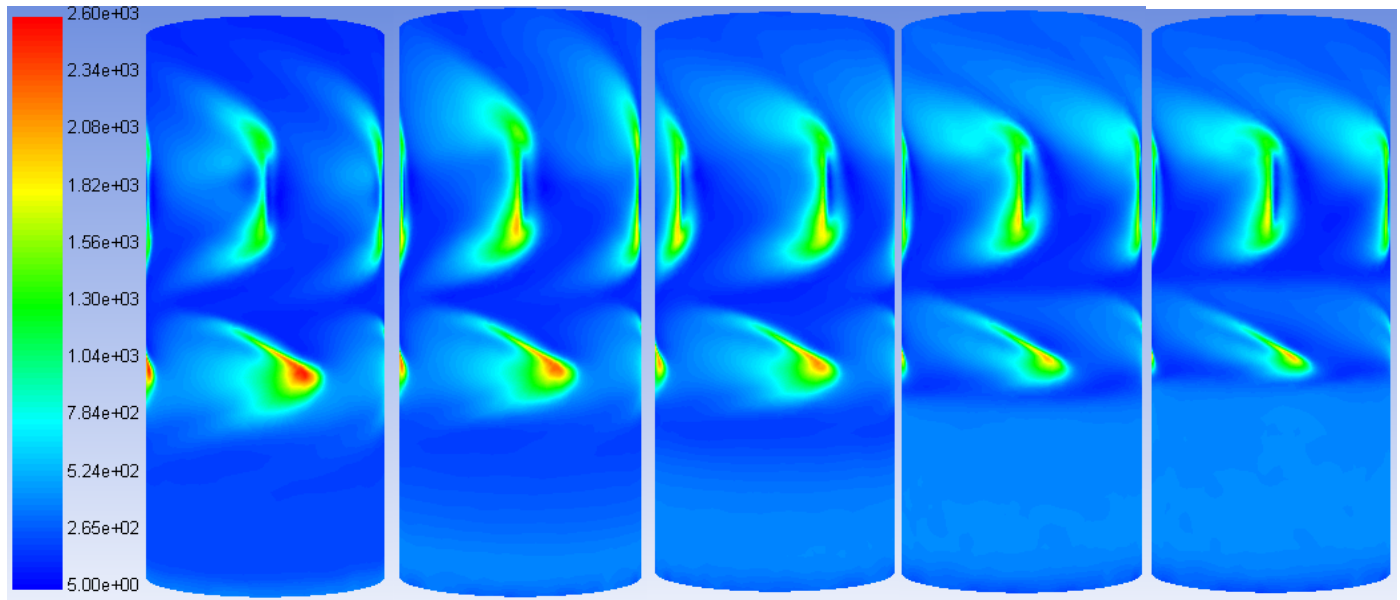

(c) Guide Vanes 5

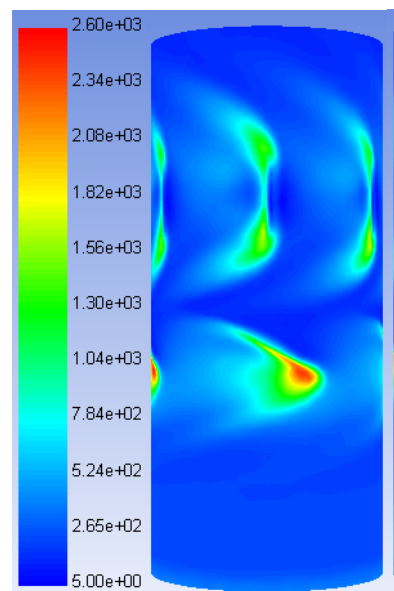

$\mathrm{Q}=1$

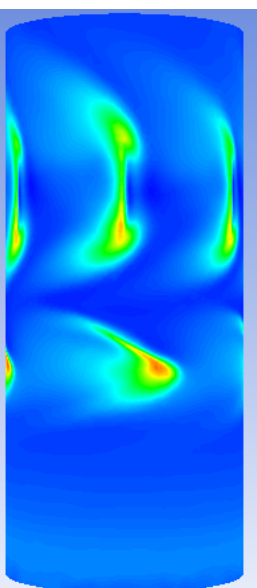

$\mathrm{Q}=2$

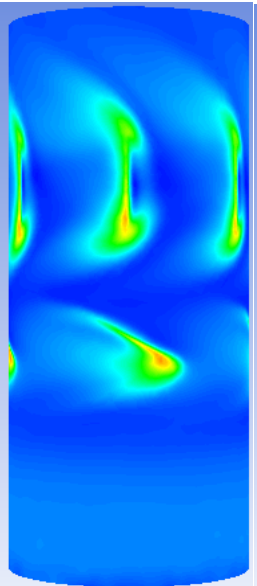

$\mathrm{Q}=3$

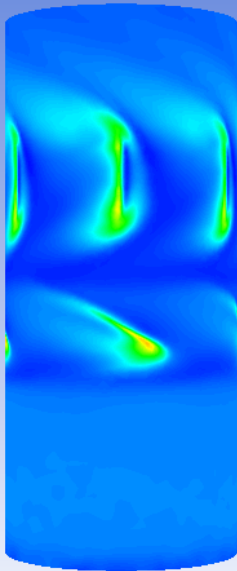

$\mathrm{Q}=4$

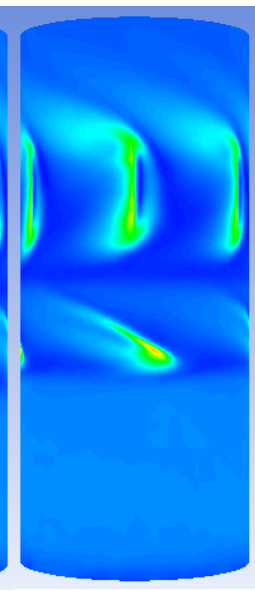

$\mathrm{Q}=5$

(d) Guide Vanes 6

Figure 10. Variations in wall shear stress (Pa) in the pump.

\section{ANALYSIS OF VELOCITY MAGNITUDE IN THE PUMP}

Figure 11 indicates the flow velocity vectors variations inside the axial pump. It is observed that the velocity magnitude increases from the area of pump suction to the discharge area. The minimum velocity magnitude take place at the inlet part of the pump and maximum velocity magnitude happened at the guide vanes part near the tip vanes. Comparing between the pictures in this figure it can be seen that under the different guide vanes the distribution of velocities is appearing have the same trend. The flow fields round and near the impeller blades and guide vanes are in disorder condition due to the swirl flow generation in this region. The flow turbulence near the vanes are more than 
disorder state as compared to the impeller blades. The formed of separation flow and vortex were occurred at the back of guide vane and the tail blade. Also, the flow separation was happened at the facing water region. There is no significant different velocities for various vanes the maximum velocity magnitude for design flow at different vanes 6, 5, 4 and 3 are $16.01,16.02,16.03$ and $16.022(\mathrm{~m} / \mathrm{s})$ respectively.

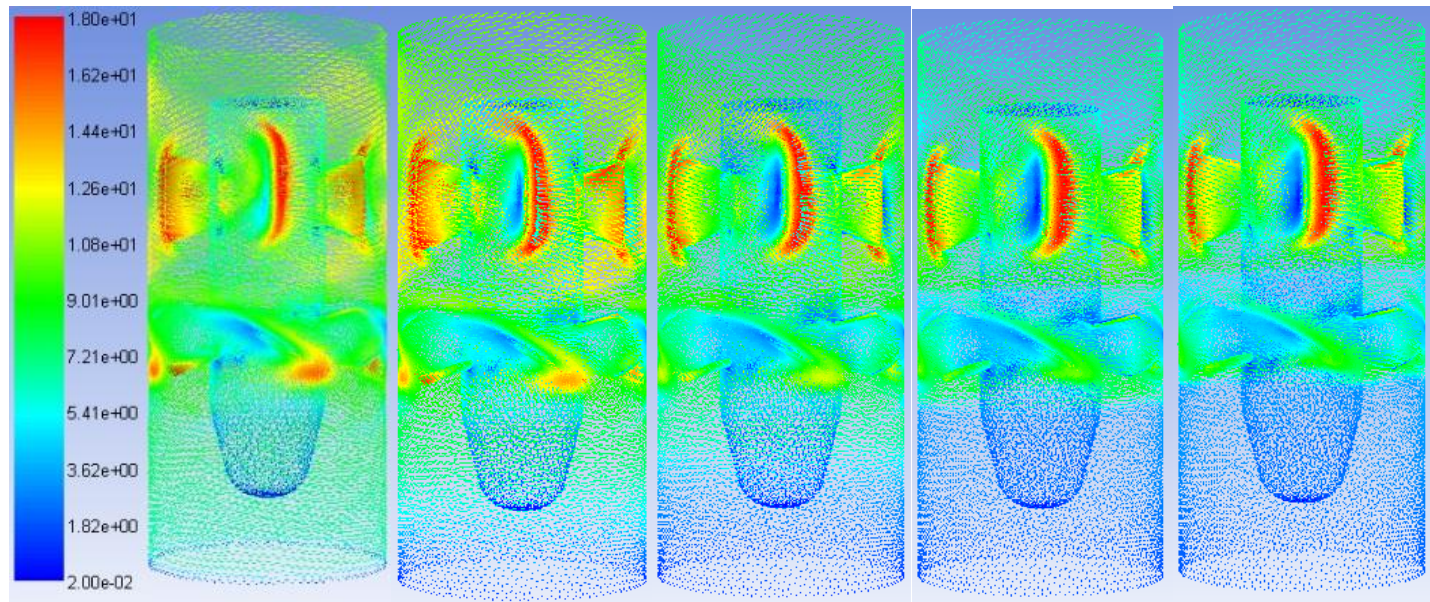

(a) Guide Vanes 3

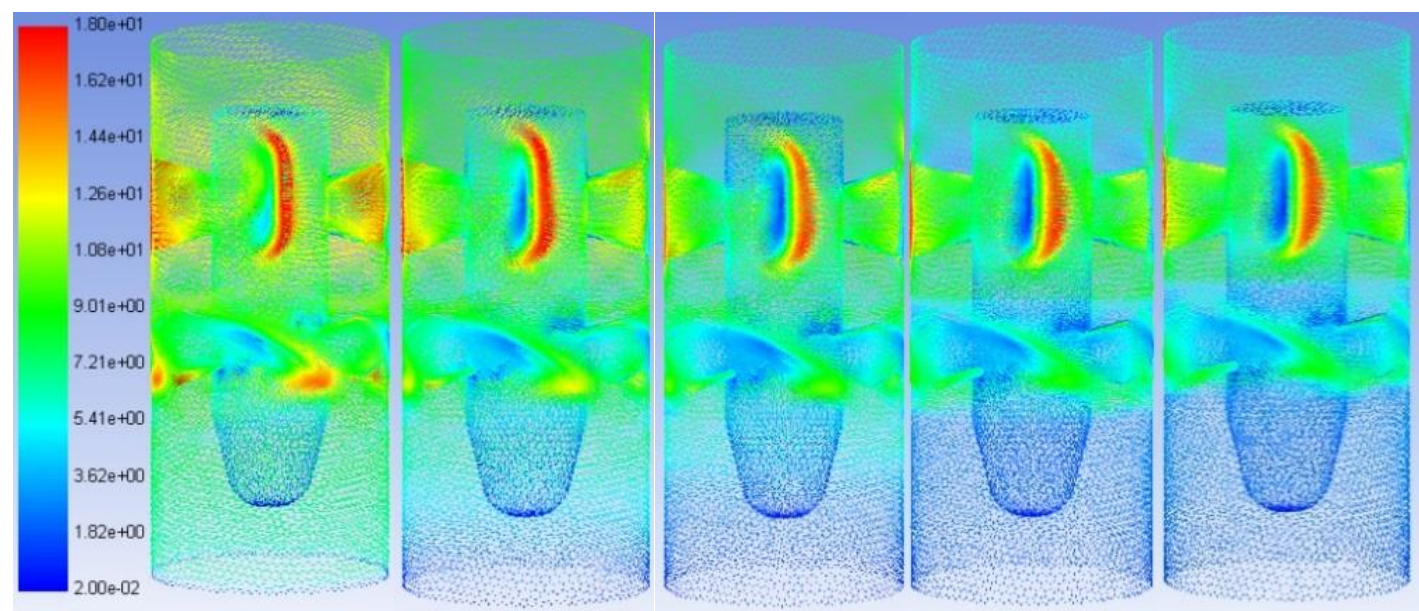

(b) Guide Vanes 4

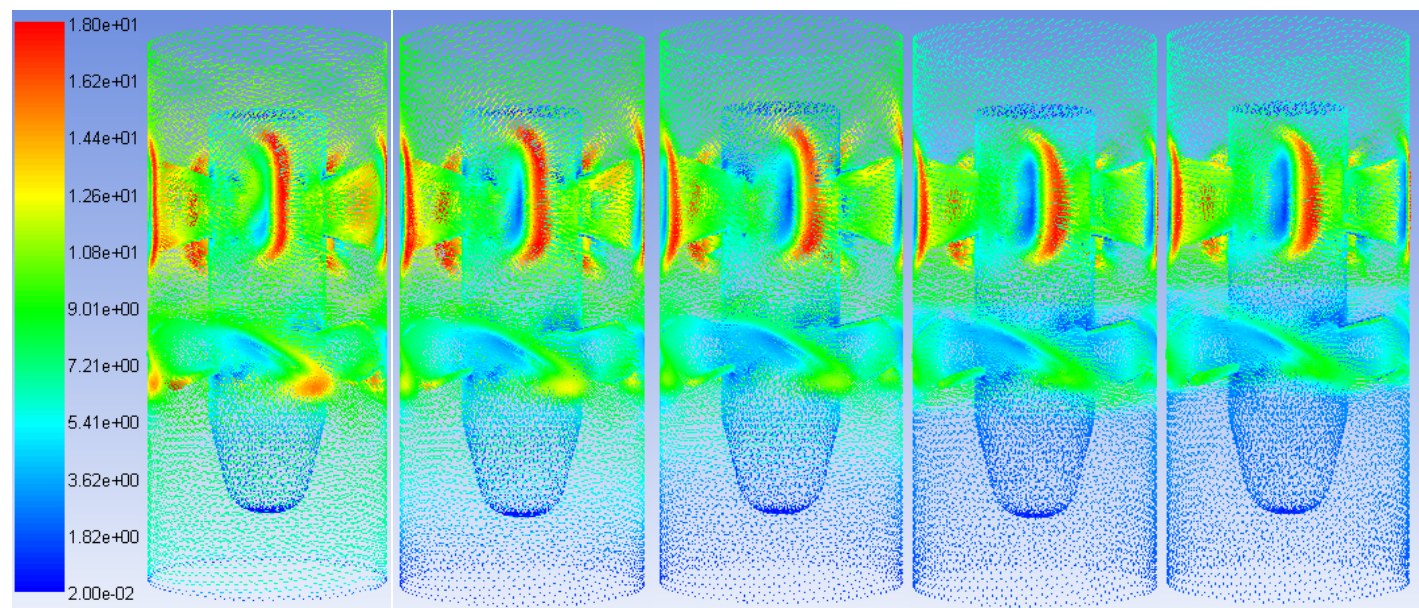

(c) Guide Vanes 5 


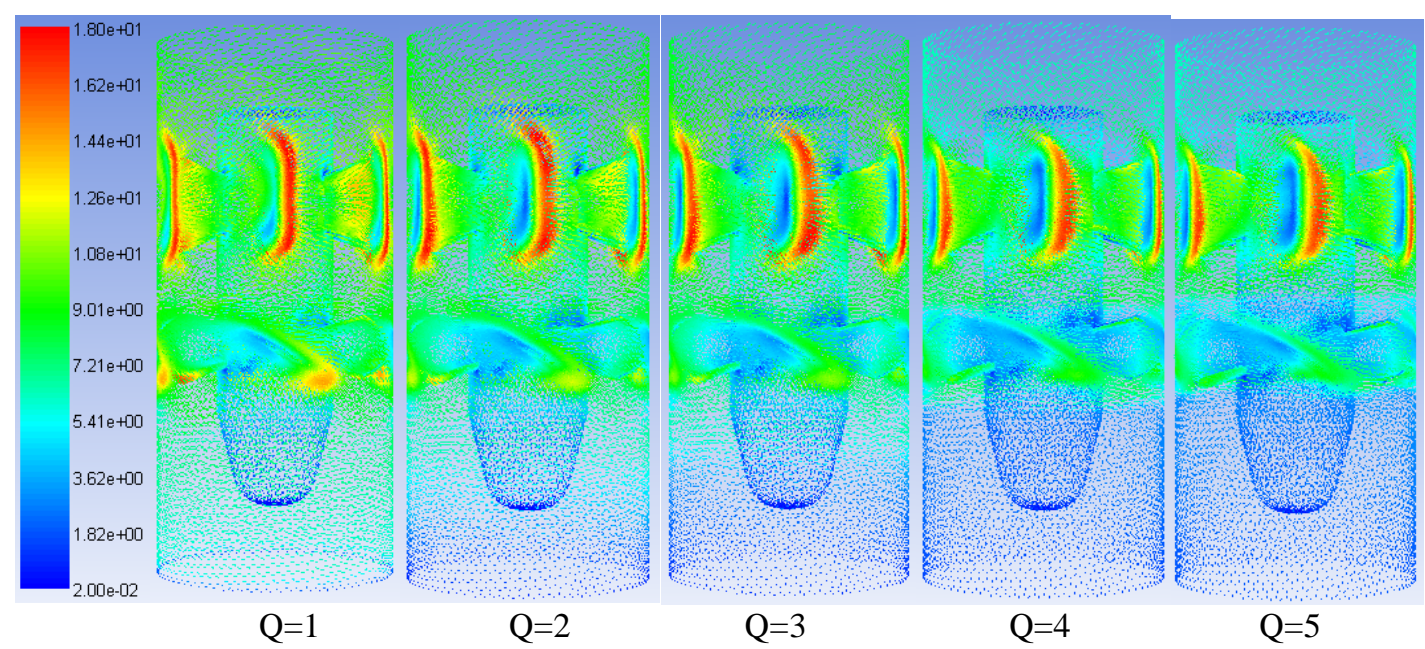

(d) Guide Vanes 6

Figure 11. Velocity magnitude variations $(\mathrm{m} / \mathrm{s})$ in the pump.

\section{VARIATIONS IN STATIC PRESSURE IN THE CENTRE SECTION}

Figure 12 illustrated the flow fields (pressure distributions) in the pump. Numerical results shown that the pressure region at the discharge of the pump is higher than inlet zone. Comparing between different flow rates the pressure distributions are seeming same tendency. The flow phenomenon in the middle cross section reveals that the pressure deceases as the axial pump is worked at high mass flow for all cases under investigations. It is observed that when the pump is worked at different flows, the fields of flow in vanes are enhanced and increased the variations in pressure. Also, the outlet flow change resulting in the change of different operation conditions. which are suitable for decreasing the hydraulic losses and enhancement pump performance. Moreover, it is found that the low pressure zone exists distinct at the suction part near the impeller leading edge as well as the high pressure were near the impeller trailing edge zone at the pressure part. The reason behind that is due to interaction between the impeller and wall pipe as well as between the outlet guide vanes. Furthermore, the distribution of pressure in the latter region was is not as regular.

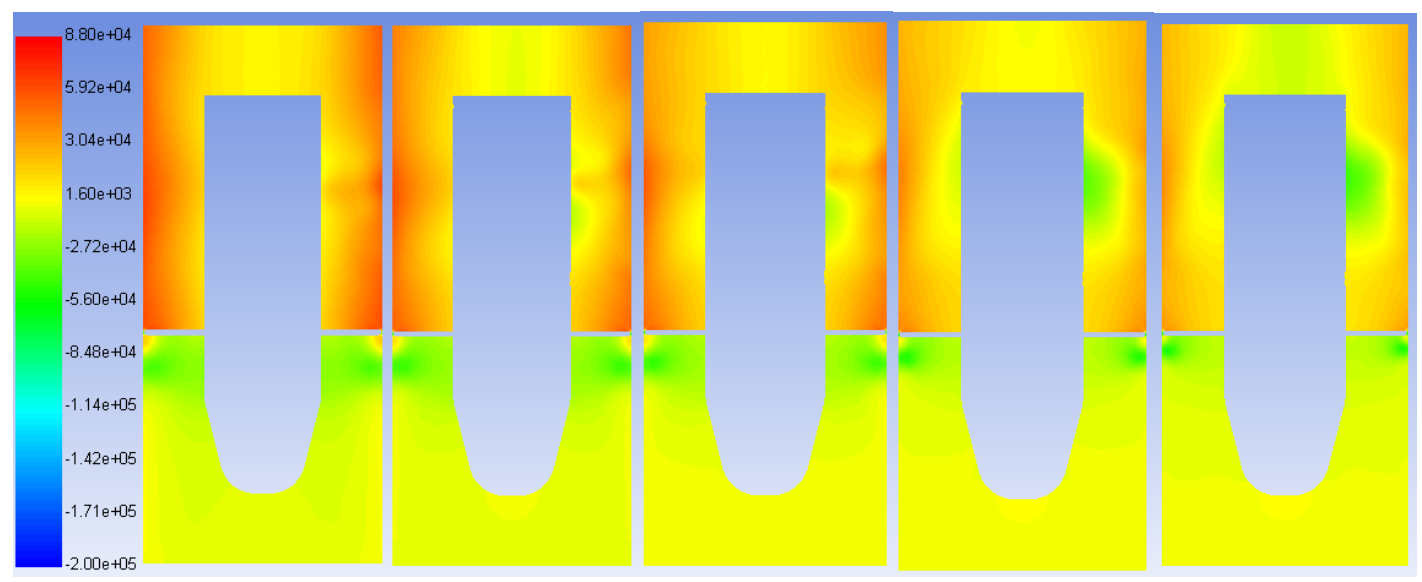

(a) Guide Vanes 3 


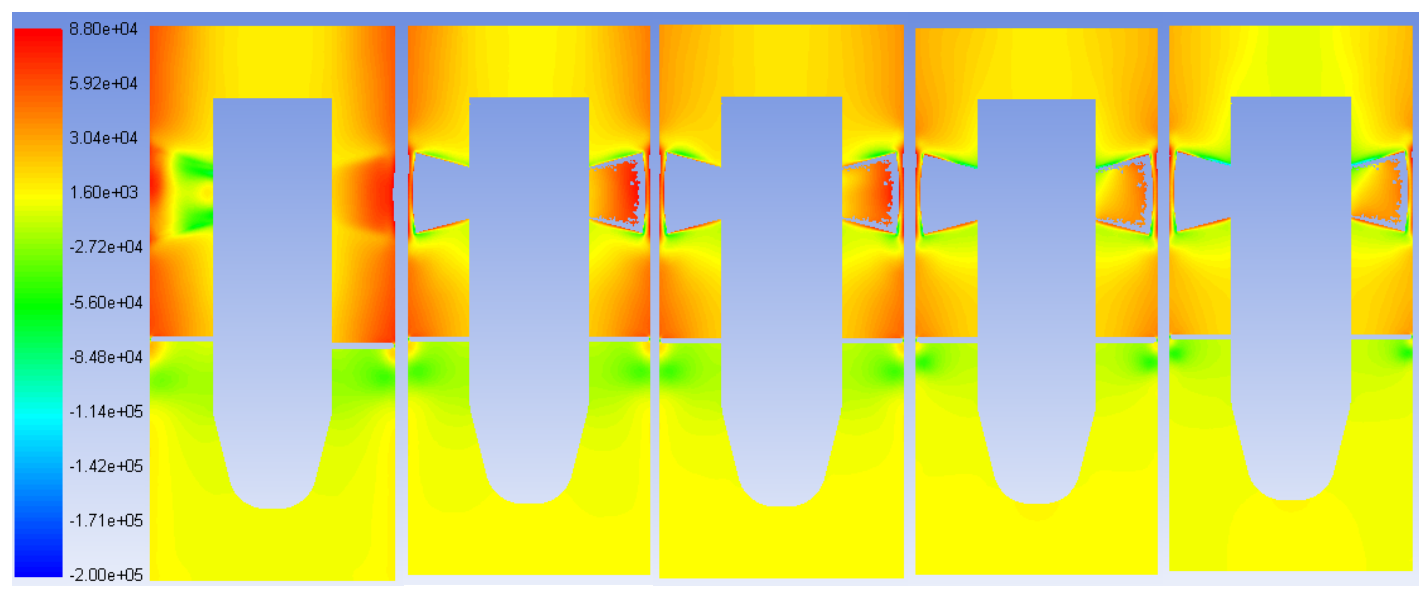

(b) Guide Vanes 4

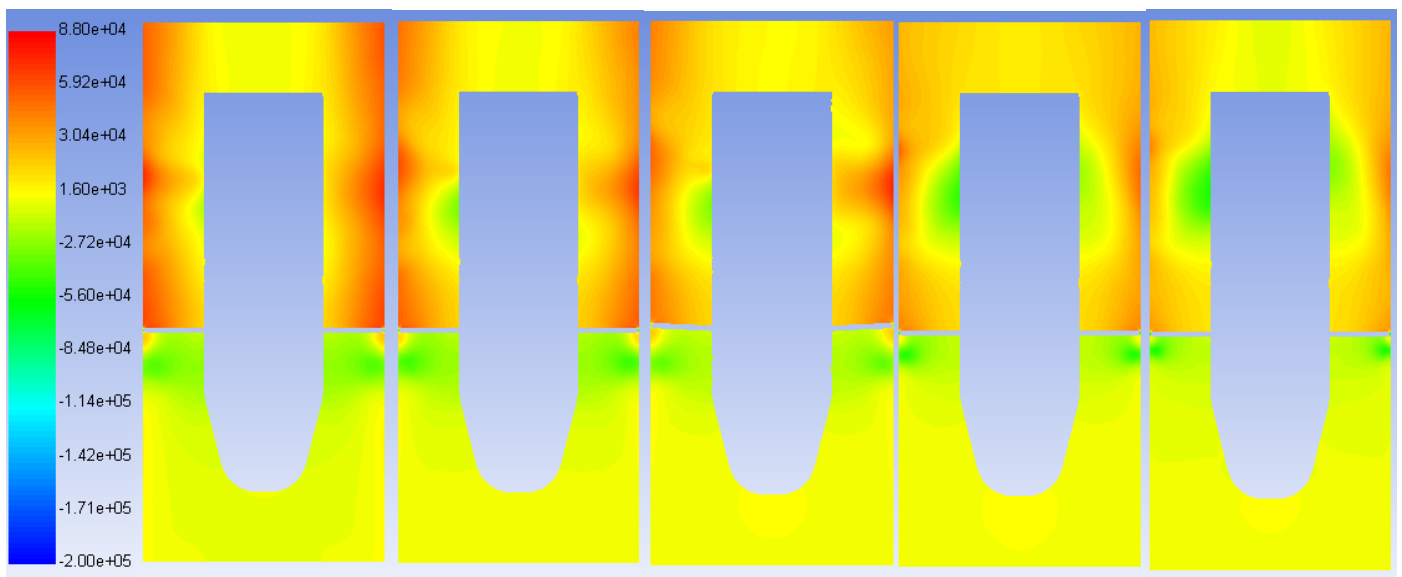

(c) Guide Vanes 5

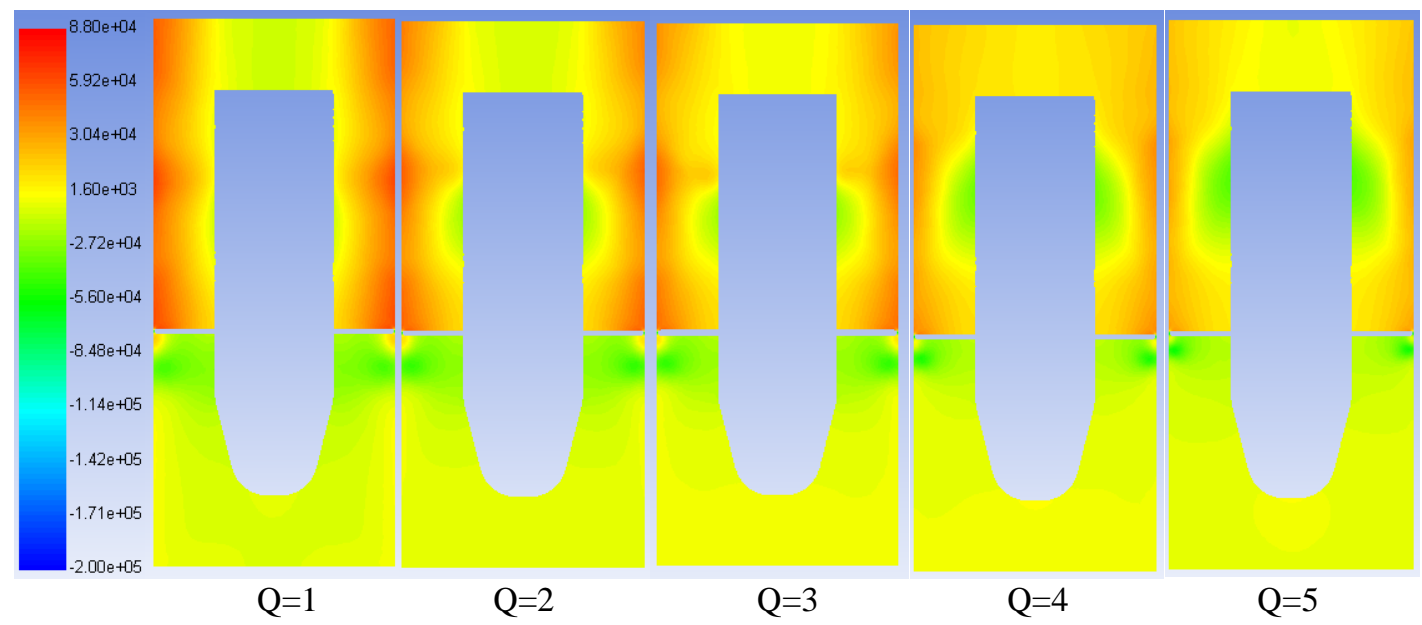

(d) Guide Vanes 6

Figure 12. Distribution in static pressure variations $(\mathrm{Pa})$ in a pump.

\section{DISTRIBUTION IN STATIC PRESSURE IN THE CENTRE SECTION}

The distribution of static pressure in cross section area can be simulated to analyses mechanism of the internal fluid. Figure 13 demonstrated the field of static pressure in the meddle cross section of the pump at several flow rates and guide vanes. As noticed that the static pressure increases across an axial flow pump at constant pump rotational speed of 3000 rpm with flow rate increases, as expected because of the flow losses in the pump. As it is found that there is no big alteration between the pressure at the inlet impeller side for all cases, due to the effect of different number of guide vanes are highly effect at after the impeller region as shown that in previous figures. Also, the maximum pressure happens at 
the outlet blade region near the tip blade area owing to the interaction in this region between both impeller blade and pipe wall.

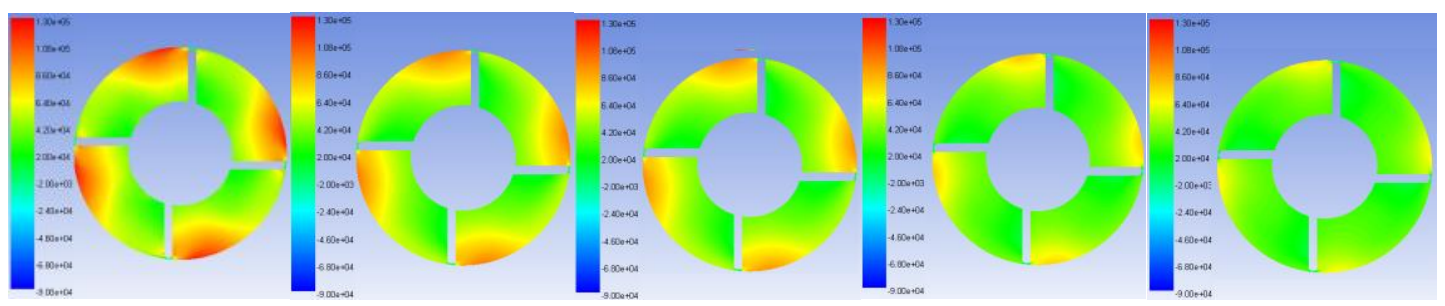

(a) Guide Vanes 3

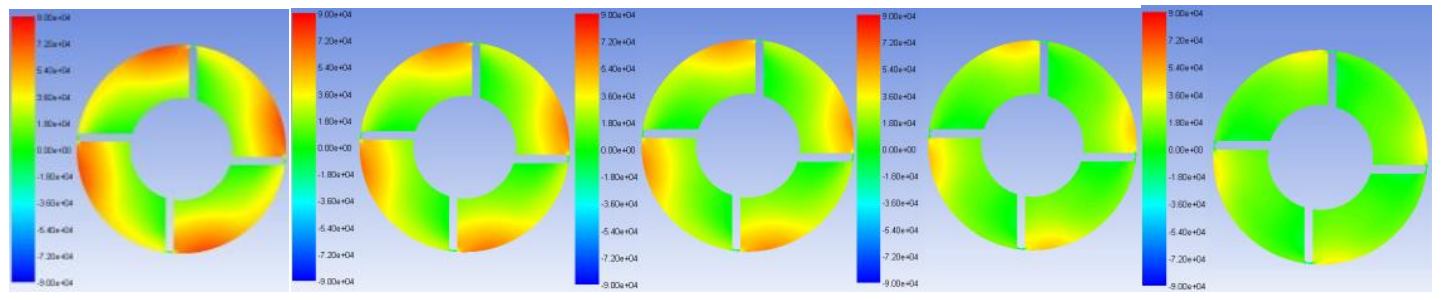

(b) Guide Vanes 4

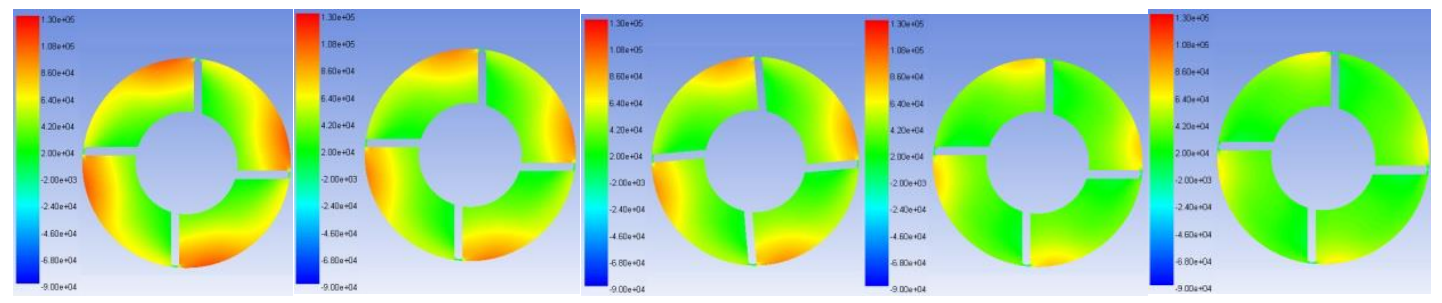

(c) Guide Vanes 5

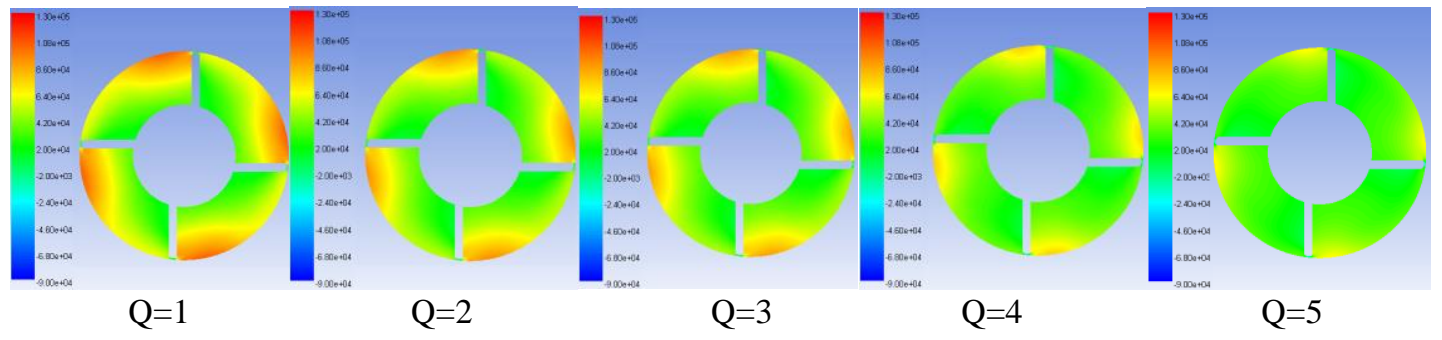

(d) Guide Vanes 6

Figure 13. Distribution in static pressure variations $(\mathrm{Pa})$.

Figure 14 depicts dynamic pressure field in the center blade impeller cross sectional-area. Results illustrated that the pressure reduces as mass flow in the pump rises. These cross section contours the pressure distribution was not regular and it increases from hub region to shroud zone. As it is seen that the variations in dynamic pressure in the blade has the same static pressure variations in previous figure it reduces when flow in the pump rises and the maximum dynamic pressure occurs nearby the tip blade region. At the high-pressure region in blade, it can be seen that the pressure was practically change under different flow rate, especially at the blade leading edge, where the pressure was high due to the incidence of high flow in this region. However, at blade impeller leading edge the pressures was low due to the flow acceleration.

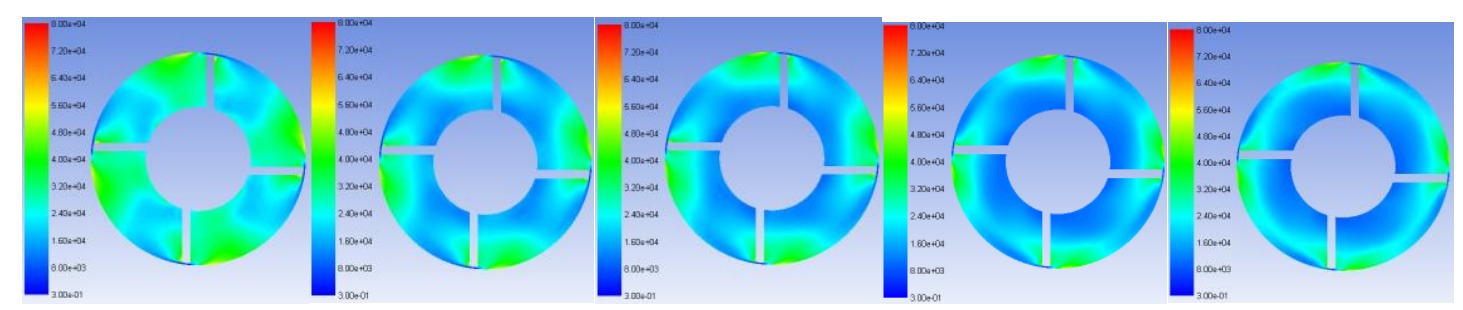

(a) Guide Vanes 3 


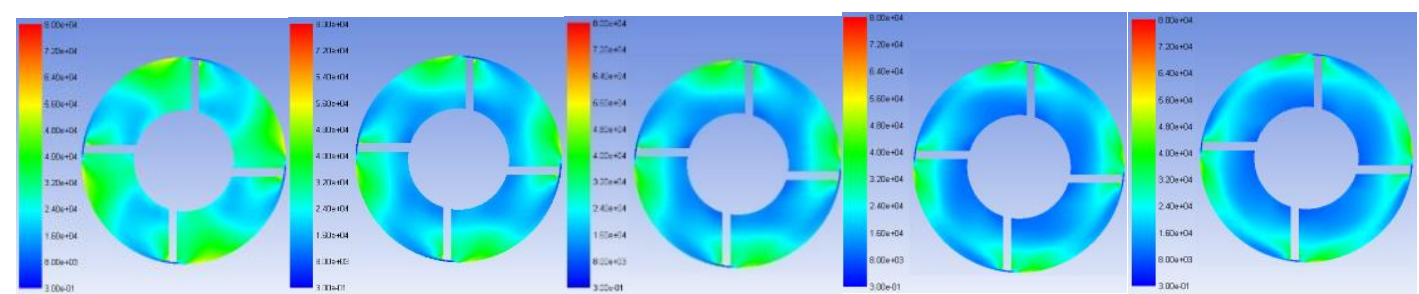

(b) Guide Vanes 4

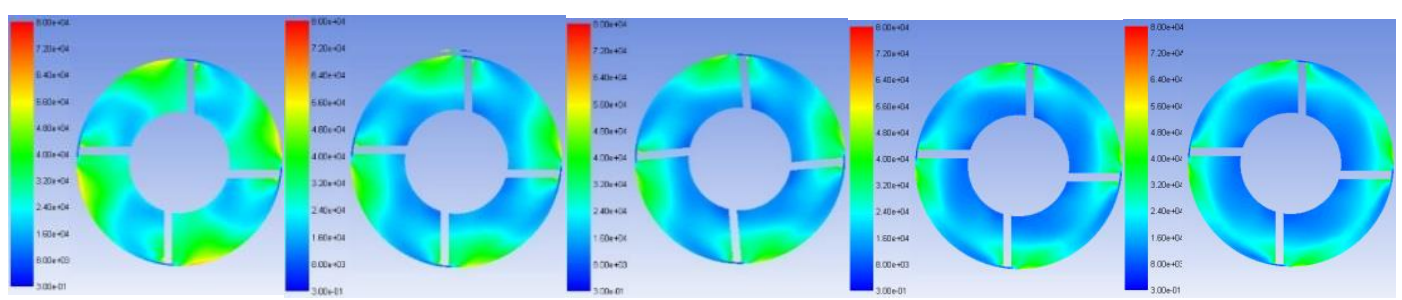

(c) Guide Vanes 5

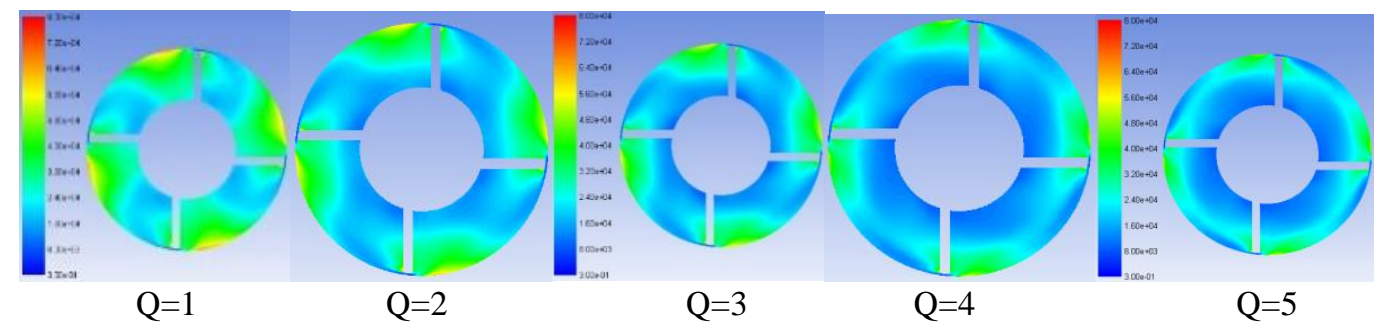

(d) Guide Vanes 6

Figure 14. Distribution of static pressure $(\mathrm{Pa})$ at various flows and vanes.

Average pressure at 49 monitoring points in the centre impeller blade cross section regions and between the impeller and vane then after guide in the pump are collected by transient numerical calculated simulation as illustrated in Figure 15.

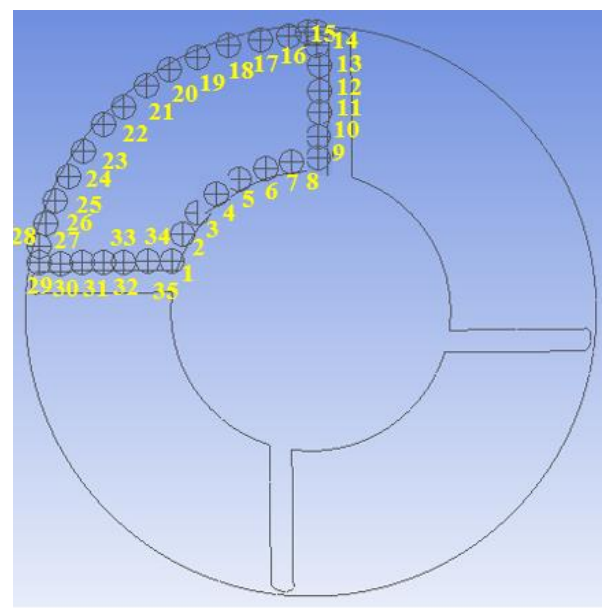

(a)

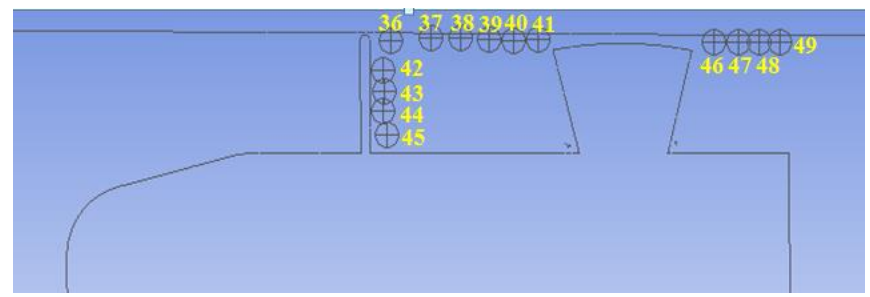

(b)

Figure 15. Distributions of monitoring points at the: (a) impeller blade and (b) cross section of axial pump. 
Figure 16 (a) depicts the comparison between average pressure in time domain and pressure amplitudes under different number of guide vane for monitoring point start from 1 to 8 in the impeller blade and at design pump flow rate. Results demonstrated that the number of guide vanes have an influence on average pressure of impeller blade in the axial pump. Also, the pressure at different monitoring point almost changes and increases from point 1 to 8 at the hub, the maximum pressure was occurred at point 8 . Additionally, the average pressure increases as number of guide vanes increases for all monitoring point under considerations. Moreover, the average percentage pressure differences in this figure between vanes $6,5,4$ and 3 are around by $7.55,13.36$ and $38.49 \%$ respectively.

Figure 16 (b) depicts the comparison between average pressure and pressure amplitudes for monitoring points from 9 to 14 at right impeller blade zone. As it is noticed that the pressure in this area increases start points from 9 to 14 the high pressure happens at this monitoring point because of position of this point near the tip blade region. Moreover, the pressure increases as guide vane increases. It can be concluded from above analyses the guide vane has high influence on inner flow and pump performance. In addition, the average percentage pressure differences between vanes 6, 5, 4 and 3 are about $4.12,8.54$ and $16.40 \%$ respectively.

Figure 16 (c) depict the pressure at the outlet impeller blade for the monitoring points from 15 to 29 . As noted that the pressure starts to decrease from points from 15 to 29 the high pressure happens at monitoring points 16,15 and 14 this due to the positions of these points are near the high interaction flow at the tip blade. The average percentage pressure differences in this figure between vanes $6,5,4$ and 3 are about 3.18, 9.46 and $13.62 \%$.

Figure 16 (d) depicts the monitoring points from 30 to 35 at left blade region. The results revealed that pressure in this region also decreases and the maximum pressure is happened at point 30 due to the same above reason in previous figure. The minimum pressure was occurred at point 35 because of the position this point nearby low pressure region and in this area of blade can lead to cause the occurrence of cavitation. Moreover, the average percentage pressure differences in this figure between vanes $6,5,4$ and 3 are around $2.43,16.25$ and $22.25 \%$ respectively.

Figure 16 (e) depicts the monitoring points from 36 to 41 for the region between the impeller and guide vanes. As seen that pressure in this region starts slightly decrease and then it increases due to reach to the high interaction area near guide vanes tip region. Moreover, the average percentage pressure differences in this figure between vanes $6,5,4$ and 3 are $4.11,9.25$ and $13.17 \%$ respectively.

Figure 16 (f) depicts the monitoring points from 42 to 45 for the region after the impeller blade. As indicted that the pressure in this area also decrease and it reach to minimum pressure at point 45 for all cases under investigations. Average percentage pressure differences in this figure between vanes 6, 5, 4 and 3 are 4.37, 13.12 and 19.30\%.

Last Figure $16(\mathrm{~g})$ depicts the monitoring points from 46 to 49 for the region after the guide vanes. It can be noticed that the pressure in this area decrease and the minimum pressure was at point 49 because of the position of this point was far from the high-pressure area near tip vanes. Moreover, the average percentage pressures are $6.18,22.67$ and $30.37 \%$ respectively.

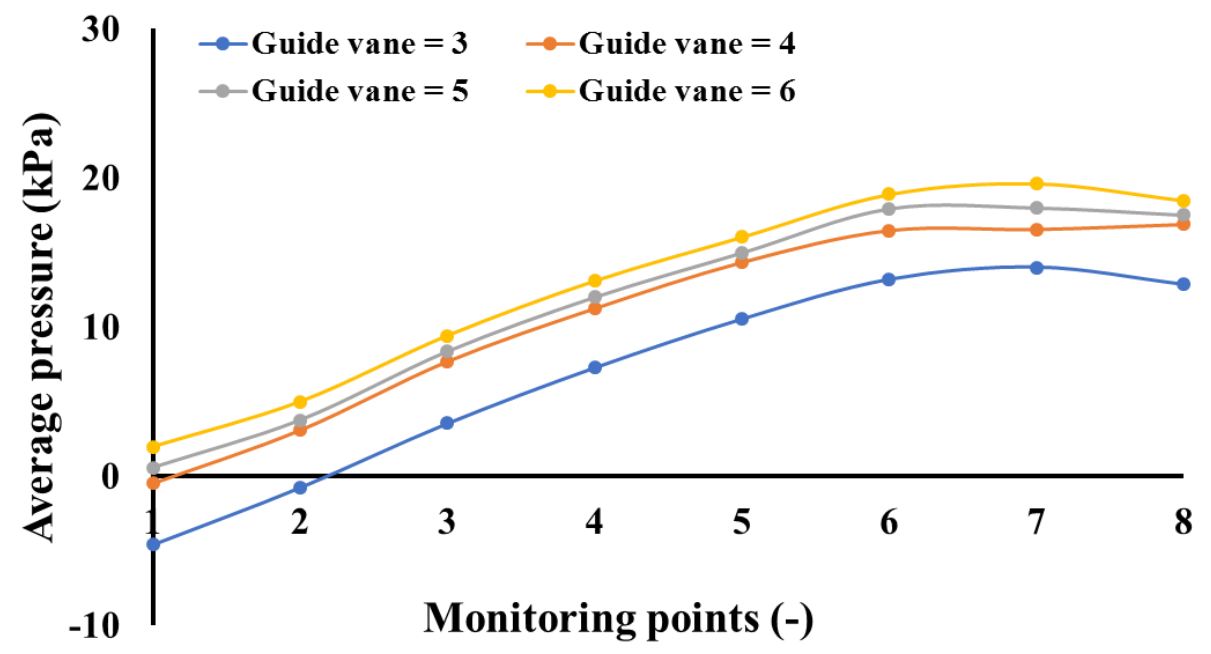

(a) 


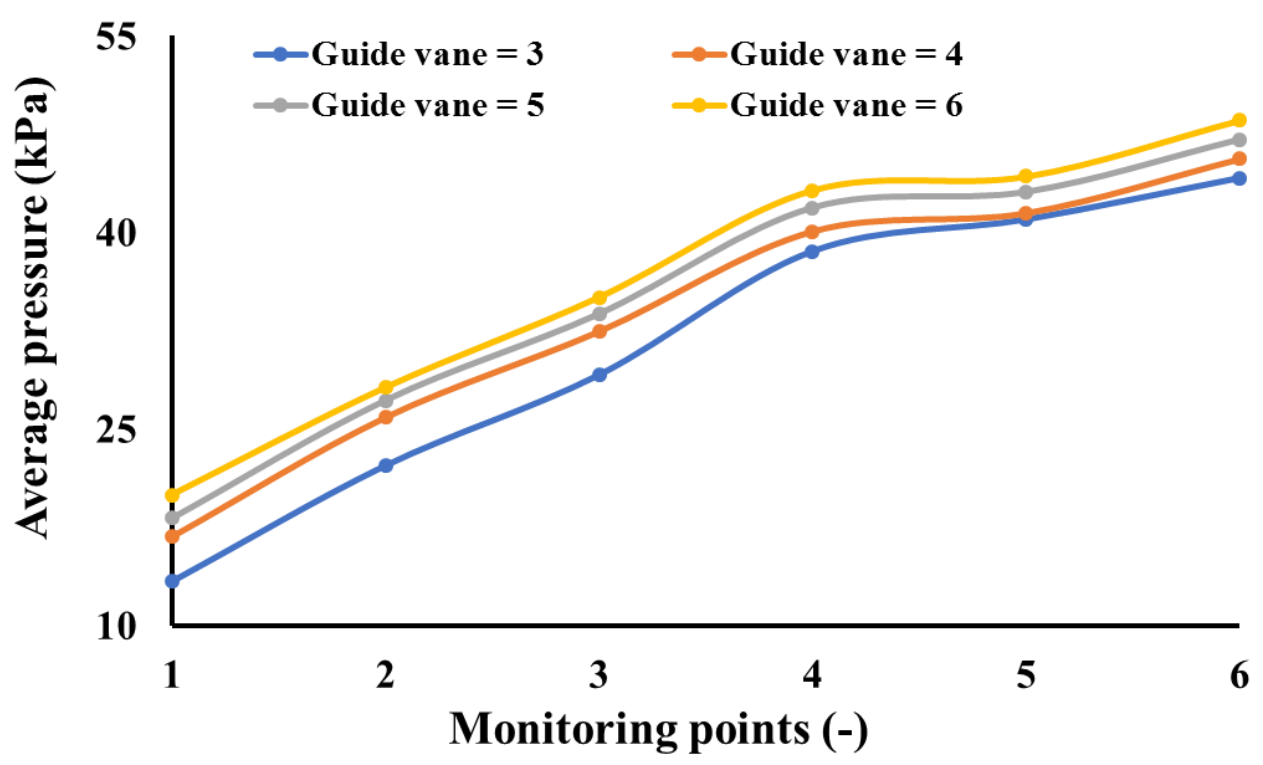

(b)

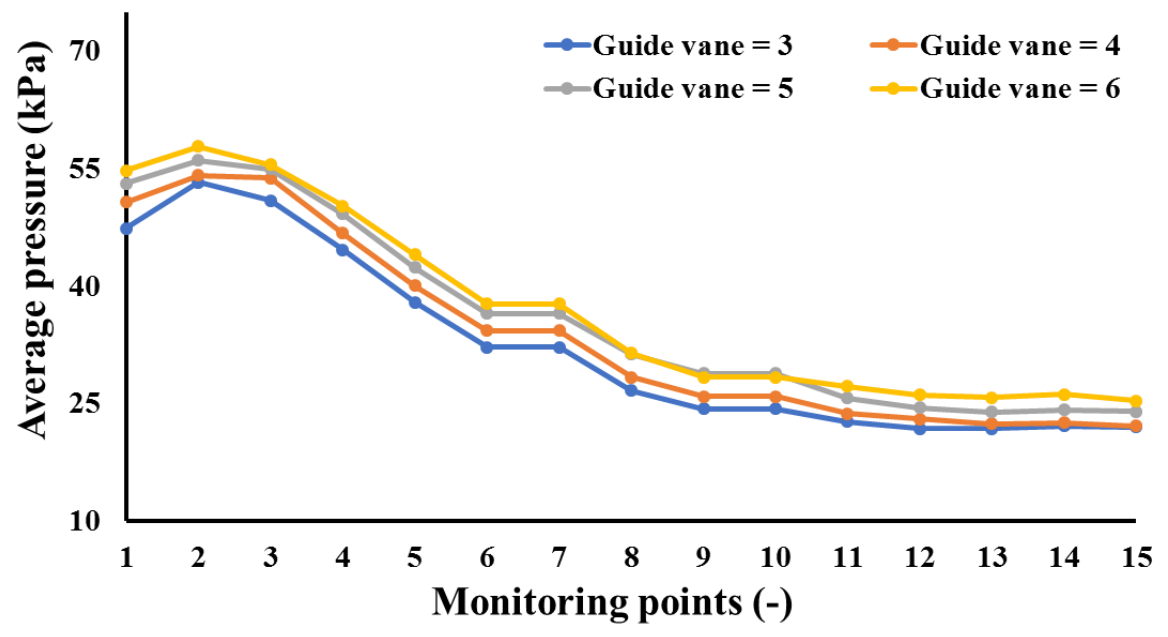

(c)

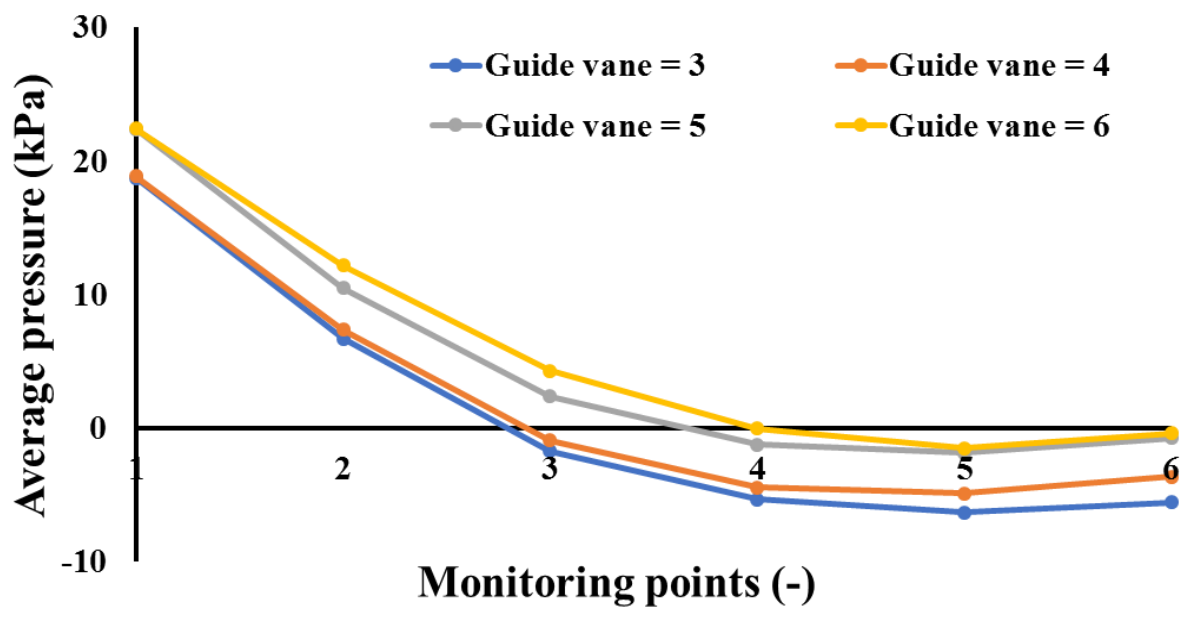

(d) 


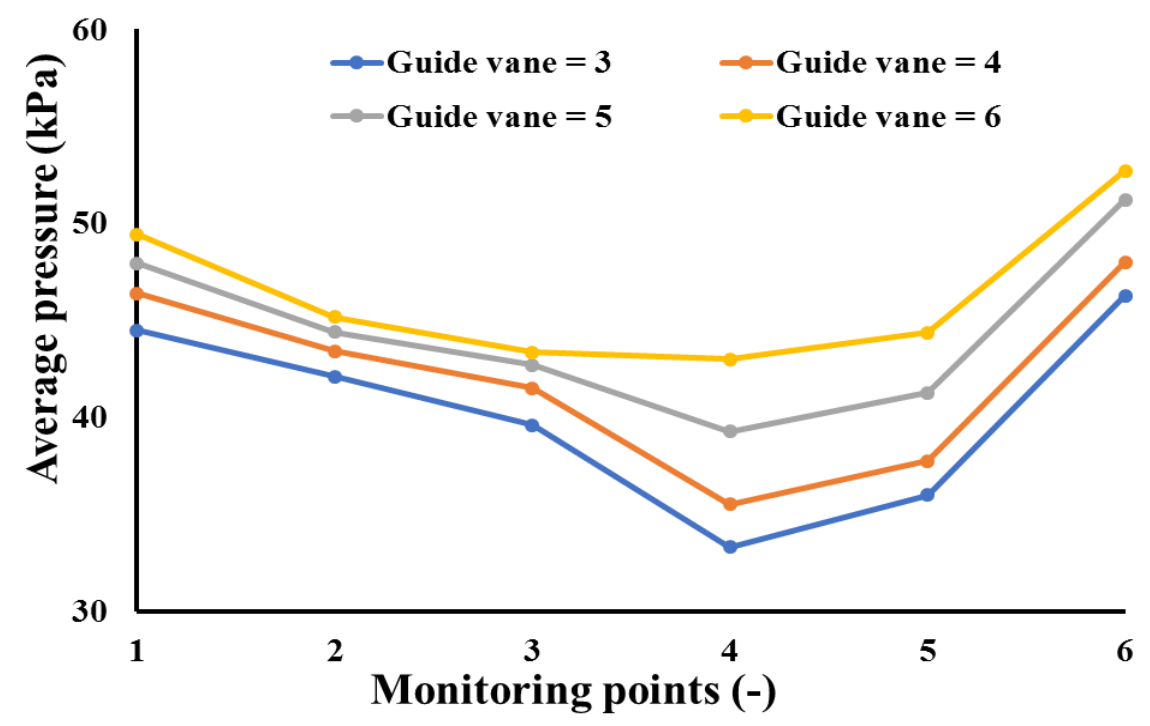

(e)

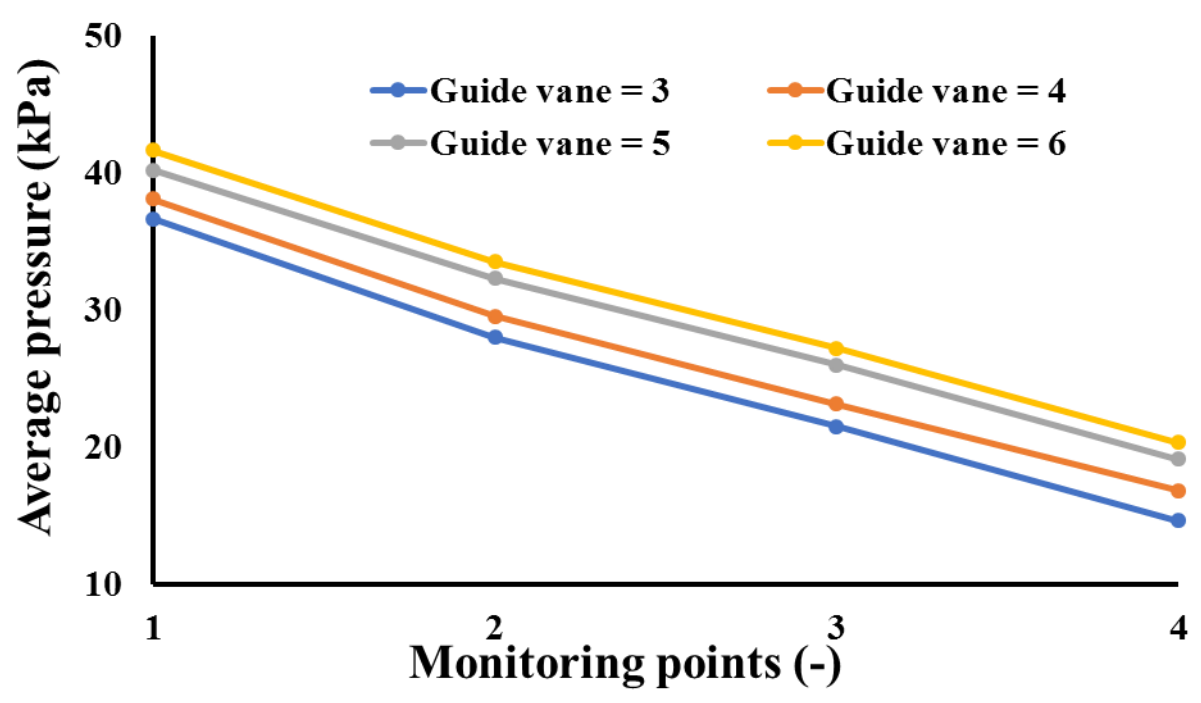

(f)

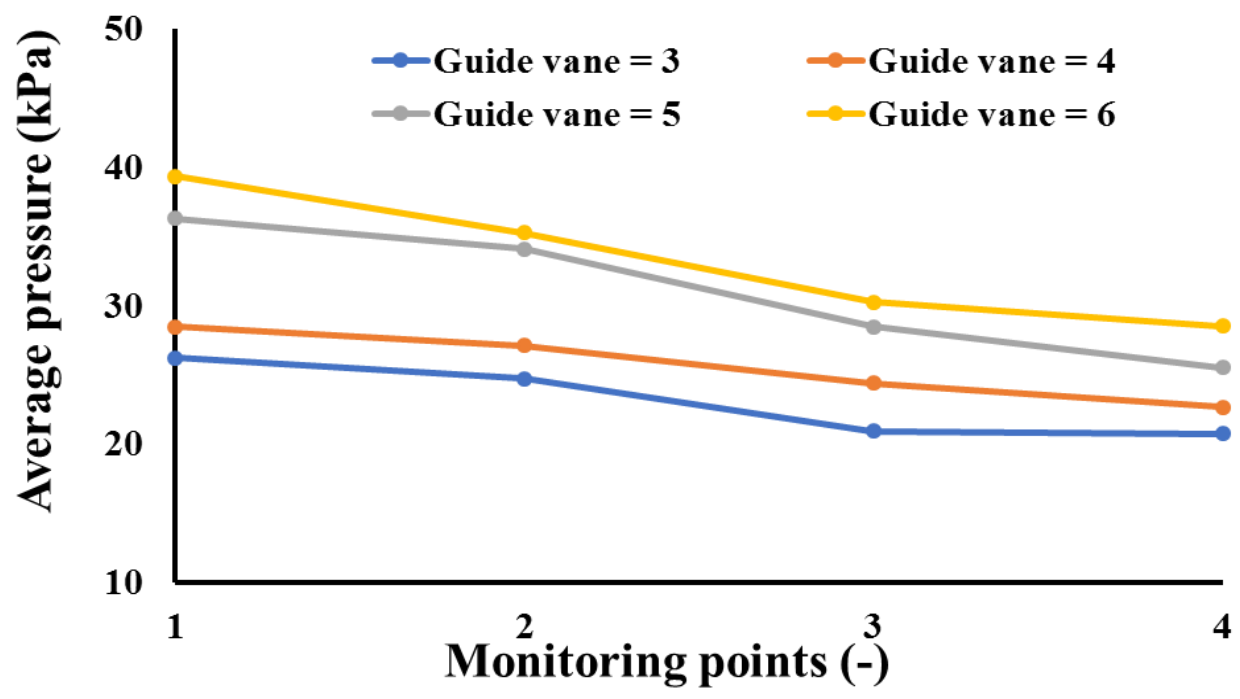

(g)

Figure 16. Distributions of monitoring points results in the blade and cross section of an axial pump. 


\section{CONCLUSIONS}

The influences of different vanes on the characteristic of flow and performance prediction in axial pump are analyses and can be found the following conclusions: The comparison between both numerical results and experimental data shown that there is small difference. The results found that the number of guide vanes are important aspect affecting internal flow for instance pressure, TKE, velocity, shear stress and velocity magnitude. When the rotating of axial impeller that leads to occur two regions first one is formed higher pressure region at outlet impeller pressure surface. The second zone is formed near low pressure at inlet impeller suction part at this region as the pressure deceases lower than vapour water pressure can leads to cavitation occurrence due to low pressure area then that causes more unstable internal flow. Also, when the guide vanes increase that leads the flow passages between the vanes is narrowed and hence the internal flow velocity increases. Moreover, the quantitative analysis shown that when the vane increases that leads to the pressure differences across the axial pump increases and hence the characteristic performance of pump increases. Static pressure differences for design flow between different vanes 6, 5, 4 and 3 are around by 4.01, 5.66 and $6.74 \%$ respectively. When the flow rate increases the TKE variations decreases and the high area of TKE happened at impeller tip blade and at tip guide vane blades. The maximum TKE for design flow between different vanes $6,5,4$ and 3 are about by $0.02,0.19$ and $2.35 \%$ respectively. Additionally, the velocity increases from the inlet of the pump to the outlet area. Minimum velocity take place at the suction side and maximum velocity happened at the guide vanes part near the tip vanes. The flow fields round and near the impeller blades and guide vanes are in disorder condition. Based on the research findings it can be observed that the impeller with number of vane 6 was the best vane. Furthermore, the results indicate that, using guide vane can increasing the pump efficiency. Pressure differences across the pump at variety mass flow for vane 6 is higher than other vanes 3,4 and 5 by $14.13,11.35$ and $3.85 \%$ for flow of $5 \mathrm{~L} / \mathrm{min}$. Also, the inner flow of vane is enhanced and the separation flow are reduced that leads to the pumping hydraulic efficiency also enhances therefore this type of pumps can meet the demand of different industry engineering applications.

\section{ACKNOWLEDGMENT}

The author would like to thank Mustansiriyah University (www.uomustansiriyah.edu.iq) Iraq - Baghdad for their support. Moreover, he many thank the University of Huddersfield, United Kingdom because of the model geometry was done when he was studied in this University in UK.

\section{REFERENCES}

[1] W.Shi, P. Zou, D. Zhang, and L. Zhou, "Unsteady flow pressure fluctuation of high-specific-speed mixed-flow pump," Transactions of the Chinese Society of Agricultural Engineering, 27(4), 147-152. 2011, doi: 10.3969/j.issn.10026819.2011.04.025.

[2] A. Al-Obaidi, "Experimental and Numerical Investigations on the Cavitation Phenomenon in a Centrifugal Pump". University of Huddersfield, UK, 2018, http://eprints.hud.ac.uk/id/eprint/34513.

[3] S. Varbak Neşe, O. Kilic, and T. Akinci, "Analysis of wind turbine blade deformation with STFT method," Periodical Analysis of wind turbine blade deformation with STFT method, 2012.

[4] A.R. Al-Obaidi, " Investigation of effect of pump rotational speed on performance and detection of cavitation within a centrifugal pump using vibration analysis," Heliyon, 5(6), e01910 doi: 10.1016/j.heliyon.2019.e01910.

[5] M.Miyabe, A. Furukawa, H. Maeda, and I.. Umeki, "Investigation of internal flow and characteristic instability of a mixed flow pump," In ASME 2009 fluids engineering division summer meeting (pp. 315-321). American Society of Mechanical Engineers Digital Collection, doi: 10.1115/fedsm2009-78277.

[6] A.R. Al-Obaidi, "Monitoring the performance of centrifugal pump under single-phase and cavitation condition: A CFD analysis of the number of impeller blades," Journal of Applied Fluid Mechanics, 12(2), 445-459, 2019, doi: 10.29252/jafm.12.02.29303.

[7] C. Li, and B. van Esch," Blade interaction forces in a mixed-flow pump with vaned diffuser," in ASME Fluids Engineering Division Summer Meeting. 2009. American Society of Mechanical Engineers Digital Collection, 2009, pp. 165-173.

[8] A.R. Al-Obaidi, "Experimental investigation of the effect of suction valve opening on the performance and detection of cavitation in the centrifugal pump based on acoustic analysis technique," Archives of Acoustics, 44, 2019. 44, doi: 10.24425/aoa.2019.126352.

[9] R. Zhu, H. Yan, Q. Fu, and A. Yang, " Numerical calculation of characteristics of tubular pump internal pressure pulsation," Shuili Fadian Xuebao (Journal of Hydroelectric Engineering), 31(1), 220-225. 2012.

[10] D. Zhang, S Shi, W. D., Chen, B., and X. F. Guan, " Unsteady flow analysis and experimental investigation of axial-flow pump," Journal of hydrodynamics, 22(1), 35-43. 2010, doi: 10.1016/s1001-6058(09)60025-1.

[11] D. L. Zariatin, D. Rahmalina, E. Prasetyo, A. Suwandi, and M. Sumardi, " The effect of surface roughness of the impeller to the performance of pump as turbine pico power plant," Journal of Mechanical Engineering and Sciences, 13(1), 4693-4703, 2019, doi: 10.15282/jmes.13.1.2019.24.0394.

[12] H. Yaguchi, T. Mishina, and K. Ishikawa," A new type of magnetic pump with coupled mechanical vibration and electromagnetic force," Journal of Mechanical Engineering and Sciences, 13(3), 5212-5227, doi: 10.4236/eng.2014.69051. 
[13] D. Adanta, E. Quaranta, and T. Mahlia," Investigation of the effect of gaps between the blades of open flume Pico hydro turbine runners," Journal of Mechanical Engineering and Sciences, 13(3), 5493-5512, 2019, doi: 10.15282/jmes.13.3.2019.18.0444.

[14] A.R. Al-Obaidi, " Effects of different turbulence models on three-dimensional unsteady cavitating flows in the centrifugal pump and performance prediction," International Journal of Nonlinear Sciences and Numerical Simulation, 20(3-4), 487-509, 2019, doi: 10.1515/ijnsns-2018-0336.

[15] A.R. Al-Obaidi, " Numerical Investigation of Flow Field Behaviour and Pressure Fluctuations within an Axial Flow Pump under Transient Flow Pattern Based on CFD Analysis Method," in Journal of Physics: Conference Series. 2019. IOP Publishing, doi: 10.1088/1742-6596/1279/1/012069.

[16] A. R. Al-Obaidi, and H. Towsyfyan, " An Experimental Study on Vibration Signatures for Detecting Incipient Cavitation in Centrifugal Pumps Based on Envelope Spectrum Analysis," Journal of Applied Fluid Mechanics, 12(6), 2057-2067, 2019, doi: 10.1299/jsdd.2.274.

[17] S. Toksoz, G. Coskun, C. Celik, H. S. Soyhan, E. Buyukkaya, and F. Halici, " Modeling of an HCCI engine by using CFD and detailed chemical kinetic model, "Energy Education Science and Technology Part A-Energy Science and Research, 29(1), 427432, 2012.

[18] L. Yi-bin, L. Ren-nian, and W. Xiu-yong, " The numerical simulation of unsteady flow in a mixed flow pump guide vane, " Journal of Fluid Machinery and Systems, 6(4), 200-205, 2013, doi: 10.6041/j.issn.1000-1298.2016.S0.024.

[19] Z. Qian, Y. Wang, W. Huai, and Y. Lee, " Numerical simulation of water flow in an axial flow pump with adjustable guide vanes, " Journal of mechanical science and technology, 24(4), 971-976, 2010, doi: 10.1007/s12206-010-0212-z.

[20] G. Li, E. Chen, A. Yang, Z. Xie, and G. Zhao," Effect of Guide Vanes on Flow and Vibroacoustic in an Axial-Flow Pump," Mathematical Problems in Engineering, 2018. doi: 10.1155/2018/3095890.

[21] F. Yang, H. R. Zhao, and C. Liu," Improvement of the efficiency of the axial-flow pump at part loads due to installing outlet guide vanes mechanism," Mathematical Problems in Engineering, 2016. doi 10.1155/2016/6375314:.

[22] X. Sang, and X. Zhou, " Investigation of hydraulic performance in an axial-flow blood pump with different guide vane outlet angle," Advances in Mechanical Engineering, 9(8), 1687814017715423. 2017, doi: 10.1177/1687814017715423.

[23] W. Li, W. Shi, Y. Xu, L. Zhou, and P. Zou, " Effects of guide vane thickness on pressure pulsation of mixed-flow pump in pumped-storage power station," Journal of Vibroengineering, 15(3), 1177-1185, 2013.

[24] S. Kim, Y. S. Choi, K. Y. Lee, and J. H. Kim, " Interaction of impeller and guide vane in a series-designed axial-flow pump, " In IOP Conference Series: Earth and Environmental Science (Vol. 15, No. 3, p. 032027). 2012, IOP Publishing, doi: 10.1088/1755-1315/15/3/032027,

[25] Z. Qian, F. Wang, Z. Guo, and J. Lu, " Performance evaluation of an axial-flow pump with adjustable guide vanes in turbine mode, " Renewable Energy, 99, 1146-1152, 2016, doi: 10.1016/j.renene.2016.08.020.

[26] A. R. Al-Obaidi, " Detection of Cavitation Phenomenon within a Centrifugal Pump Based on Vibration Analysis Technique in both Time and Frequency Domains, " Experimental Techniques, 1-19. 2020, doi: 10.1007/s40799-020-00362-z.

[27] A. R. Al-Obaidi, and A. A. Mohammed, " Numerical Investigations of Transient Flow Characteristic in Axial Flow Pump and Pressure Fluctuation Analysis Based on the CFD Technique, " Journal of Engineering Science \& Technology Review, 12(6). 2019, doi: 10.25103/jestr.126.09.

[28] A. R. Al-Obaidi, " Investigation of the influence of various numbers of impeller blades on internal flow field analysis and the pressure pulsation of an axial pump based on transient flow behavior, " Heat Transfer. 2020 doi: 10.1002/htj.21704.

[29] X. Song, C. Liu, and F. Yang, " Influence of the inlet vortex on axial flow pump unit. in IOP Conference Series: Earth and Environmental Science. 2019. IOP Publishing, doi: 10.1088/1755-1315/240/3/032005.

[30] X. Song, and C. Liu, " Experimental investigation of pressure pulsation induced by the floor-attached vortex in an axial flow pump, " Advances in Mechanical Engineering, 11(3), 1687814019838708, 2019, doi: 10.1177/1687814019838708.

[31] Y. Zhou, W. Hu, Y. Zhou, and Y. Zheng, " Analysis of Pressure Fluctuation and Stress Deformation in Reverse Generation of Large Vertical Axial Flow Pump, "In IOP Conference Series: Earth and Environmental Science (Vol. 237, No. 3, p. 032093). 2019, IOP Publishing, doi: 10.1088/1755-1315/237/3/032093.

[32] N. H. Mostafa, and M. Adel, " Effect of blade angle on cavitation phenomenon in axial pump, " J Appl Mech Eng, 1(112), 2, 2012, doi: 10.4172/2168-9873.1000112.

[33] A. R. Al-Obaidi, " Investigation of fluid field analysis, characteristics of pressure drop and improvement of heat transfer in three-dimensional circular corrugated pipes, " Journal of Energy Storage, 26, 101012. 2019, doi: 10.1016/j.est.2019.101012.

[34] A. R. Al-Obaidi, and A. Sharif, " Investigation of the three-dimensional structure, pressure drop, and heat transfer characteristics of the thermohydraulic flow in a circular pipe with different twisted-tape geometrical configurations, " Journal of Thermal Analysis and Calorimetry, 1-26. 2020, doi: 10.1007/s10973-019-09244-y.

[35] A. F. Nowakowski, J. C.Cullivan, R. A. Williams, and T. Dyakowski, " Application of CFD to modelling of the flow in hydrocyclones. Is this a realizable option or still a research challenge, " Minerals Engineering, 17(5), 661-669, 2004, doi: 10.1016/j.mineng.2004.01.018.

[36] H.Zhu, R.Zhang, B. Xi, and D. Hu, " Internal Flow Mechanism of Axial-Flow Pump with Adjustable Guide Vanes, " In ASME 2013 Fluids Engineering Division Summer Meeting. American Society of Mechanical Engineers Digital Collection, doi: 10.1115/FEDSM2013-16613. 